\title{
Induction of protective immunity against Mycobacterium tuberculosis by delivery of ESX antigens into airway dendritic cells
}

\author{
H Dong ${ }^{1,2,3}$, O Stanek ${ }^{4}$ F Rudilla Salvador ${ }^{1,2}$, U Länger ${ }^{1,2}$, E Morillon ${ }^{1,2}$, C Ung ${ }^{1,2}$, P Sebo ${ }^{4,5}$, \\ C Leclerc ${ }^{1,2,5}$ and L Majlessi ${ }^{1,2,5}$
}

As the Bacillus Calmette-Guérin (BCG) vaccine does not confer long-lasting protection against lung Mycobacterium tuberculosis infection, the development of more efficient vaccines is greatly needed. Here, we used mycobacterial lowmolecular weight proteins of the 6-kDa Early Secreted Antigenic Target (ESAT-6) protein family (ESX) antigens for the evaluation of a novel vaccine delivery strategy that enables versatile in vivo targeting of antigens into specialized dendritic cell (DC) subsets. ESX antigens were genetically fused to the tetramerizing core of streptavidin (SA) to form high-affinity complexes with biotin (biot)-conjugated antibodies recognizing DC surface receptors. When directed through the CD11 b or CD11C $\beta_{2}$-integrins or diverse C-type lectins, the ESX-SA:biot-antibody complexes were efficiently captured and presented on major histocompatibility complex molecules of DCs to specific T-cell receptors. Robust ESXspecific T-cell responses were induced by immunization with as little as several picomoles of ESX-SA targeted to DC subsets. Moreover, directing of TB10.4-SA to airway CD205 ${ }^{+}$cells enabled the induction of mucosal T-cell responses and provided significant protection against virulent $M$. tuberculosis.

\section{INTRODUCTION}

A promising approach to the development of novel subunit vaccines is antigen (Ag) delivery to dendritic cell (DC) subsets, which has key roles in the induction of adaptive immunity. Such vaccines could build on the knowledge of the properties of diverse DC subsets, defined according to their ontogenic origin, phenotype, and functions in different tissues. In the mouse spleen, three major DC subsets have been identified: (i) $\mathrm{CD}_{11 \mathrm{c}^{+}} \mathrm{B}^{2} 20^{+} \mathrm{BST}-2$ (bone marrow stromal cell Ag-2, $\left.\mathrm{CD}_{17}\right)^{+}$plasmacytoid DCs (pDCs), specialized in the production of type-I interferons (IFNs); (ii) $\mathrm{CD} 11 \mathrm{c}^{+} \mathrm{CD} 1 \mathrm{~b}^{-}$ $\mathrm{CD} 8_{\alpha}^{+}$DCs, with the robust ability to take up dead cells, crosspresent the derived Ags, and trigger $\mathrm{T}$ cells via interleukin (IL)- 12 p70 secretion; and (iii) $\mathrm{CD} 11 \mathrm{c}^{+} \mathrm{CD} 11 \mathrm{~b}^{+} \mathrm{CD} 8_{\alpha}^{-} \mathrm{DCs}$, considered as potent inducers of major histocompatibility complex (MHC)-II-restricted T cells. ${ }^{1}$ In mouse skin, in addition to the resident DCs of the lymph nodes, the epidermisderived CD205 (DEC205) ${ }^{\mathrm{hi}}$ Langerhans cells, CD207
(Langerin) ${ }^{+} \mathrm{CD}^{205^{\text {int }}}$ conventional dermal DCs, and a $\mathrm{CD} 207^{+} \mathrm{CD}_{103^{+}}$dermal DC populations have been identified. Only the latter crosspresent Ags to naive $\mathrm{CD} 8{ }^{+} \mathrm{T}$ cells. ${ }^{2} \mathrm{At}$ steady state, the mouse trachea contains intraepithelial $\mathrm{CD}_{11 \mathrm{~b}}{ }^{-} \mathrm{CD}_{207}{ }^{+} \mathrm{CD}_{103}{ }^{+}$DCs. Under inflammatory conditions, the submucosa of airways may contain $\mathrm{CD} 11 \mathrm{~b}^{+}$ $\mathrm{CD}_{103^{-}}$conventional DCs, able to trigger $\mathrm{CD} 4^{+} \mathrm{T}$ effectors. In the lung parenchyma, $\mathrm{CD} 11 \mathrm{c}^{+} \mathrm{CD} 11 \mathrm{~b}^{+}$and $\mathrm{CD} 11 \mathrm{c}^{+}$ $\mathrm{CD}_{11 \mathrm{~b}^{-}}$DCs are present and can migrate to the alveolar lumen or to lymph nodes. CD11 $\mathrm{c}^{\text {int }} \mathrm{CD} 11 \mathrm{~b}^{-} \mathrm{BST}^{-2^{+}} \mathrm{pDCs}$ are detectable in the lung parenchyma and produce IFN- $\alpha$ upon Toll-like receptor (TLR) triggering. ${ }^{3}$ Identification of human equivalents of mouse DC subsets represents an active field of investigation, leading to translation of knowledge generated in mice to humans to develop efficient vaccines based on $\mathrm{Ag}$ delivery to DCs.

The magnitude of the adaptive immunity and differentiation of $\mathrm{CD} 4^{+} \mathrm{T}$ cells into T-helper (Th)1, Th2, or Th17 cells are

\footnotetext{
${ }^{1}$ Institut Pasteur, Unité de Régulation Immunitaire et Vaccinologie, Paris, France. ${ }^{2}$ INSERM U1041, Paris, France. ${ }^{3}$ Jiangsu Key Laboratory of Zoonosis, Yangzhou University, Yangzhou, Jiangsu, China. ${ }^{4}$ Institute of Microbiology of the ASCR, Prague, Czech Republic. ${ }^{5} \mathrm{PS}, \mathrm{CL}$, and LM are co-senior authors. Correspondence: C Leclerc and L Majlessi (claude.leclerc@pasteur.fr and laleh.majlessi@pasteur.fr). 
dictated by DC subsets. ${ }^{4}$ Thus, mobilization of different DC populations by direct in vivo targeting of their surface receptors represents a promising strategy for the development of wellcontrolled immunization schemes. ${ }^{5}$ This strategy has been pioneered by Steinman and Nussenzweig, who used Ag coupling to Abs to target receptors on DC surfaces. ${ }^{6,7}$

Although it is largely established that DCs translate information from different surface receptors into an activation program that orients Th cell differentiation, the complete set of rules governing functions of DC subsets is not yet understood. Therefore, it remains difficult to predict which DC subsets/DC surface receptors are the most appropriate to target for optimizing protective immunity against a given pathogen. Thus, comparison of the properties of various DC subsets on the generation of specific adaptive responses may help to identify the most appropriate DC subsets likely able to confer the most protective immunity. In this respect, the strategy of directed Ag delivery to DCs remains limited by the need for tedious individual chemical coupling or genetic fusion of the immunogen of interest to each individual monoclonal antibody $(\mathrm{mAb})$, specific to any of the numerous DC surface receptors.

Here, we elaborated a versatile system enabling a combinatorial approach to identify appropriate receptor(s) for immunogen delivery into DCs. The strategy relies on genetic fusion of Ags to streptavidin (SA). The resulting Ag-SA tetramers can then be complexed in a combinatorial manner to a variety of biotin (biot)-conjugated mAbs chosen from a large panel of available $\mathrm{mAbs}$ specific for diverse DC surface receptors. This yields an inherent flexibility of choice in receptor targeting.

This system was applied to the development of a vaccine strategy against tuberculosis (TB), the major global infectious disease. The only vaccine currently in use against Mycobacterium tuberculosis is the live attenuated M. bovis BCG (Bacillus Calmette-Guérin), which cannot be used for booster immunization and does not confer long-lasting protection. ${ }^{8}$

Among the most promising $M$. tuberculosis immunogens are the low-molecular weight proteins of the 6-kDa Early Secreted Antigenic Target (ESAT-6) protein family (ESX), ${ }^{9}$ which are secreted by the type-VII secretion system and are known for their marked immunogenicity and protective potential in ethnically different human populations. Moreover, the presence of T-cell effectors specific to such proteins directly correlates to the natural protection in humans. ${ }^{10,11}$

We used here the DC-targeting strategy for selecting the most appropriate DC subsets or DC surface receptors for efficient capture/endocytosis/presentation of ESX Ags by MHC molecules in vivo and induction of protective $\mathrm{T}$-cell responses. Directing the TB10.4 ESX Ag to airway CD205 ${ }^{+}$cells then enabled induction of mucosal T-cell responses and protection against $M$. tuberculosis.

\section{RESULTS}

\section{Mycobacterial ESX-SA fusion proteins}

The complete sequences of mycobacterial ESX Ags ESAT-6 (ESX A), CFP-10 (ESX B), or TB10.4 (ESX H) were fused to the
$\mathrm{N}$ terminus of the tetramerization core (residues 13-139) of SA. Soluble Ag-SA tetramers were produced and purified close to homogeneity (Figure 1a) as described previously. ${ }^{12}$ The capacity of $\mathrm{Ag}$-SA fusions to assemble into tetramers was preserved, which enabled their strong binding to biot-mAbs because of the high $\left(K_{\mathrm{d}} \sim 1 \times 10^{-15} \mathrm{M}\right)$ affinity of the tetrameric SA for biot. ${ }^{13}$

\section{Ab-mediated binding of ESX-SA to DC surface receptors promotes Ag endocytosis}

We evaluated the binding of ESAT-6-SA tetramers to DCs through biot-mAbs specific to various DC surface receptors. Conventional bone marrow (BM)-DCs were pre-incubated at $4^{\circ} \mathrm{C}$ with biot-mAbs specific for CD11b or CD11c $\beta_{2}$-integrins, or for MHC-II or DCIR-2 C-type lectins before the addition of ESAT-6-SA. As detected by anti-ESAT-6 mAb, the ESAT-6-SA displayed a marked binding to the surface of biot-mAb-treated DCs compared to cells pre-incubated with biot-control immunoglobulin $\mathrm{G}(\mathrm{IgG})$ (Figure 1b). After $3 \mathrm{~h}$ incubation at $37^{\circ} \mathrm{C}$, the ESAT-6-specific surface fluorescence intensity was substantially reduced. This suggested that ESAT- 6 was endocytosed by the targeted DCs. pBM-DCs (Figure 1b, right) or lung DCs (Supplementary Figure S1 online) pre-incubated with biot-anti-BST-2 or biot-anti-CD205 mAb, respectively, and then with ESAT-6-SA also showed binding of ESAT-6 at $4^{\circ} \mathrm{C}$, followed by its internalization at $37^{\circ} \mathrm{C}$. Therefore, ESAT-6SA was delivered specifically to DC surface receptors by the selected biot-mAbs and endocytosed via $\mathrm{CD} 11 \mathrm{~b}$ or CD11c, DCIR-2, MHC-II, BST-2, or CD205.

\section{Delivery of ESX Ags into the MHC-II pathway}

We investigated the capacity of different Ag-presenting cells to which ESX Ag-SA were delivered via CD11b, CD11c, MHC-II, or BST-2 to present Ag-derived epitopes to specific T cells. BMDCs pre-incubated with biot-anti-CD11b, -CD11c or -MHCII, and then with various concentrations of ESAT-6-SA, were able to present ESAT-6 to the I-A ${ }^{\mathrm{b}}$-restricted, ESAT-6:1-20specific NB11 T-cell hybridoma or to polyclonal anti-ESAT-6 T cells from M. tuberculosis-infected mice (Figure 1c). pBM-DCs or BM-M $\phi$, to which ESAT-6-SA was delivered, respectively, via $\mathrm{BST}-2$ or CD11b, were able to stimulate NB11 hybridoma cells (Figure 1d). BM-DCs $\left(\mathrm{H}-2^{\mathrm{d}}\right)$, to which TB10.4-SA was delivered via CD11b or MHC-II, also presented TB10.4 (Figure 1e) to the I-A ${ }^{\mathrm{d}}$-restricted, TB10.4:74-88-specific 1H2 T-cell hybridoma. This demonstrates that ESX Ags delivered to the surface of conventional DCs, pDCs, or M $\phi$ via CD11b, CD11c, MHC-II, or BST-2 gain access to the MHC-II machinery.

\section{In vivo delivery of Ag-SA to $\mathrm{DC}$ subsets allows $\mathrm{Ag}$ presentation}

We evaluated the in vivo specificity of ESX Ag-SA delivery to DC subsets as well as internalization and efficiency of their presentation. For the initial in vivo assessment of the strategy, the intravenous (i.v.) model was chosen, which, unlike the lungs or dermis, offers the unique possibility to target distinct splenic DC subsets and does allow to obtain sufficient numbers of 
a
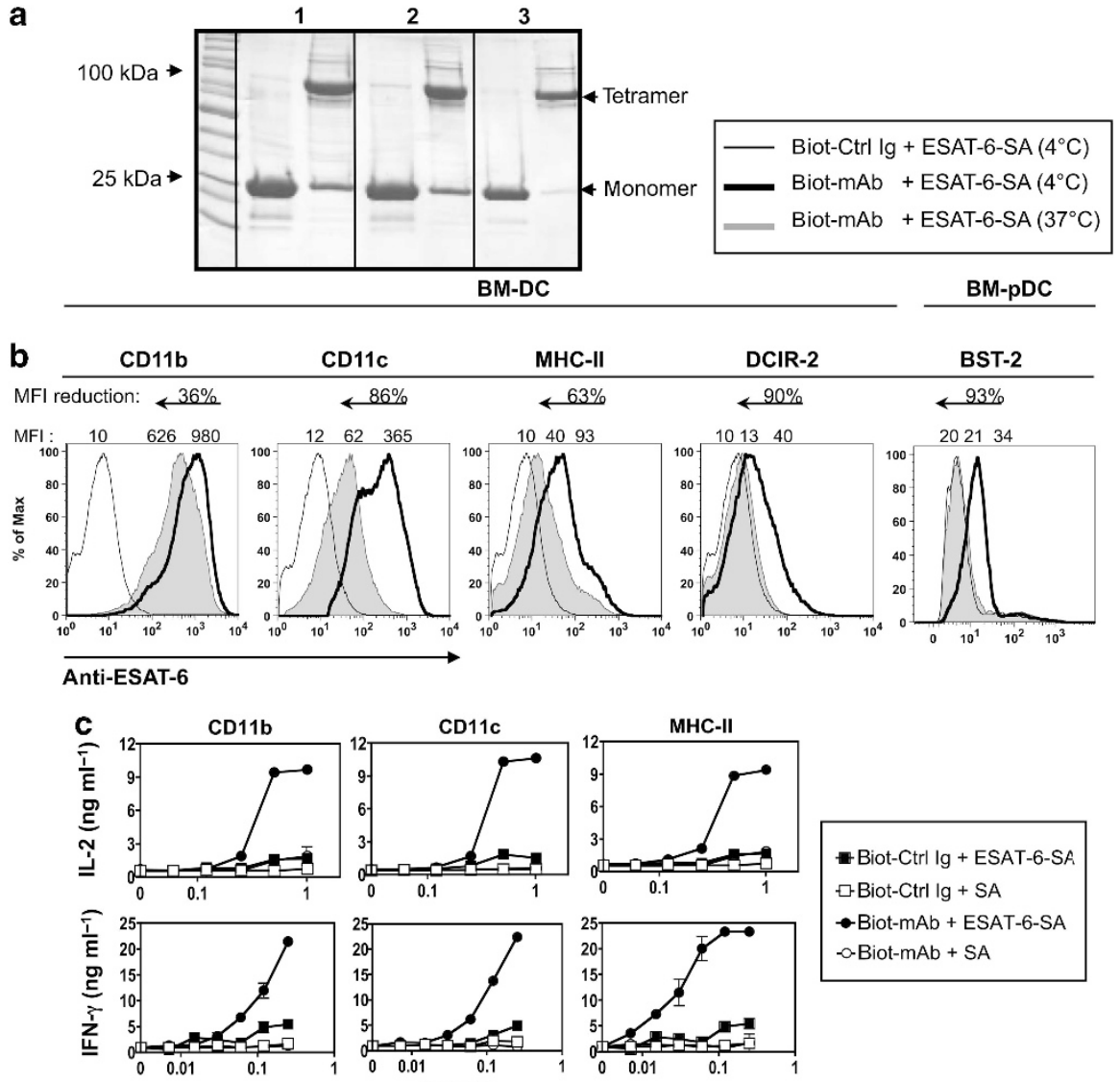

- Biot-Ctrl Ig + ESAT-6-SA

ㅁ-Biot-Ctrl lg + SA
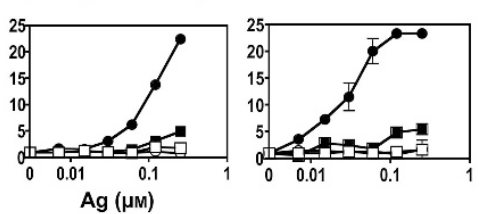

- Biot-mAb + ESAT-6-SA

$\sim$ Biot-mAb + SA
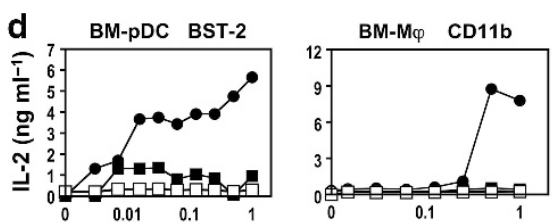

- Biot-Ctrl Ig + ESAT-6-SA

ㅁ Biot-Ctrl lg + SA

- Biot-mAb + ESAT-6-SA

o-Biot-mAb + SA
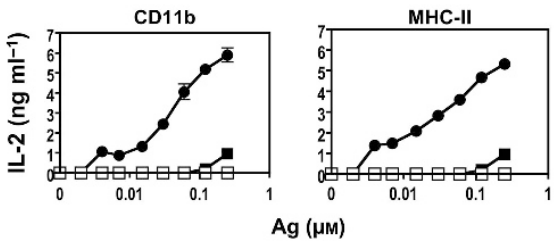
-Biot-Ctrl lg + TB10.4-SA
마 Biot-Ctrl lg + SA
- Biot-mAb + TB10.4-SA

- - Biot-mAb + SA

Figure 1 Analysis of antibody (Ab)-mediated delivery of low-molecular weight proteins of the 6-kDa Early Secreted Antigenic Target (ESAT) protein family-streptavidin (ESX-SA) fusion proteins to dendritic cell (DC) surface receptors and delivery to the major histocompatibility complex (MHC)-II machinery. (a) Sodium dodecyl sulfate-polyacrylamide gel electrophoresis (SDS-PAGE) of (1) TB10.4-SA (24.5 kDa), (2) CFP-10-SA $(24.5 \mathrm{kDa})$, and (3) ESAT-6-SA $(23.5 \mathrm{kDa})$ fusion proteins. SA with ESX-SA monomers were obtained by heating samples for $5 \mathrm{~min}$ at $100^{\circ} \mathrm{C}$. ESX-SA tetramers were stable in sample buffer at room temperature. In all, $10 \mu \mathrm{g}$ of each protein sample were loaded and separated on $15 \%$ Tris-Tricine SDS-PAGE gels stained with Coomassie Blue. (b) In vitro binding of ESAT-6-SA delivered to conventional bone marrow (BM)-DCs (left) or plasmacytoid BM (pBM)-DCs (right) by biotin (biot)-monoclonal Abs (mAbs) specific to different DC surface receptors. Cells were first incubated at $4{ }^{\circ} \mathrm{C}$ with biot-control (Ctrl) immunoglobulin G (IgG) or biot-mAbs specific to DC markers before incubation with ESAT-6-SA. Cells were then kept at $4^{\circ} \mathrm{C}$ or incubated at $37^{\circ} \mathrm{C}$ for $3 \mathrm{~h}$ to evaluate ESAT-6 internalization. The surface ESAT- 6 signal was detected by FP $647 \mathrm{H}^{-a n t i-E S A T-6}$ mAb. ESAT- 6 signal mean fluorescence intensities (MFIs) are indicated at the top of each peak, and percentages of MFI reduction at $37^{\circ} \mathrm{C}$ are indicated above each histogram. Sequential incubation of DCs with biot-mAbs and then with ESX-SA or their incubation with pre-formed ESX-SA-biot-mAb complexes led to similar level of binding (not shown). (c) MHC-II-restricted presentation of ESAT-6 by BM-DCs delivered with ESAT-6-SA via different biot-mAbs. BM-DCs $\left(\mathrm{H}-2^{\mathrm{b}}\right)$ were incubated at $4^{\circ} \mathrm{C}$ with biot-control IgG or biot-mAbs specific to diverse DC surface receptors, washed and incubated at $4^{\circ} \mathrm{C}$ with various concentrations of ESAT-6-SA or SA alone. Cells were then washed and co-cultured with ESAT-6-specific, I-A ${ }^{b}$ restricted NB11 T-cell hybridoma cells (c, top) or with Thy-1.2+ ${ }^{+}$splenocytes from $M$. tuberculosis-infected C57BL/6 mice (c, bottom). Interelukin (IL)-2 (c, top) or interferon (IFN)- $\gamma$ (c, bottom) produced by T cells was assessed in the co-culture supernatants at 24 or $72 \mathrm{~h}$, respectively. (d) MHC-II-restricted presentation of ESAT- 6 by BM-derived plasmacytoid DCs (pDCs) or BM-M $\phi$ delivered with ESAT-6-SA complexed to biot-mAbs specific to BST-2 (bone marrow stromal cell Ag-2) or CD11b, as evaluated by NB11 T-cell hybridoma. (e) Presentation of TB10.4 by BM-DCs $\left(\mathrm{H}-2^{d}\right)$ following delivery to $\mathrm{CD} 11 \mathrm{~b}$ or $\mathrm{MHC}$-II molecules, as detected by I-A ${ }^{\mathrm{d}}$-restricted $1 \mathrm{H} 2 \mathrm{~T}$-cell hybridoma. The results are representative of at least three independent experiments. Ag, antigen. 
$\mathrm{T}$ cells for a detailed analysis of the induced responses. Mice were injected i.v. with $500 \mathrm{pmol}(=10 \mu \mathrm{g})$ per mouse of ESAT6-SA, complexed to biot-anti-CD11b mAb or to biot-control IgG at a molar ratio of 2:1, together with $25 \mu \mathrm{g}$ of the TLR3 agonist poly(cytidylic-inosinic) (poly I:C), a synthetic analog of double-stranded RNA, the adjuvant used in further immunizations. At different time points postinjection, spleen low-density cells were analyzed for the presence of ESAT-6-SA at the surface of DC subsets (Figure 2a). ESAT-6-SA binding was detectable $3 \mathrm{~h}$ postinjection, only at the surface of $\mathrm{CD} 11 \mathrm{c}^{+}$ $\mathrm{CD} 8_{\alpha}^{-}\left(\mathrm{CD}_{11 \mathrm{~b}^{+}}\right) \mathrm{DCs}$, and not on $\mathrm{CD} 11 \mathrm{c}^{+} \mathrm{CD} 8_{\alpha}^{+}$ $\left(\mathrm{CD} 11 \mathrm{~b}^{-}\right)$DCs. ESAT-6-SA was barely detectable at the surface of DCs $24 \mathrm{~h}$ postinjection, suggesting the internalization of the bound ESAT-6-SA. In parallel, CD11c-eYFP mice were injected with $500 \mathrm{pmol}(=10 \mu \mathrm{g})$ per mouse of ESAT-6-SA complexed to biot-anti-CD11c mAb or to biot-control IgG. Only CD11c-eYFP cells from the former stained positively for ESAT-6-SA at $6 \mathrm{~h}$, and to a lesser extent at $24 \mathrm{~h}$ (Figure 2b), showing the specificity of binding followed by the internalization of the delivered $\mathrm{Ag}$.

We then studied the in vivo Ag presentation by the targeted DC after injection of Ag-SA:biot-mAb complexes. BALB/c mice were injected i.v. with $50 \mathrm{pmol}(=1 \mu \mathrm{g})$ per mouse of TB10.4-SA complexed at a molar ratio of $1: 1$ to control biot-IgG or biot-mAbs specific to CD11b, CD11c, DCIR-2, or CD209 (DC-SIGN). The molar ratio of 1:1 used for the formation of the complexes left, on average, one free biot molecule conjugated per molecule of $\mathrm{mAb}$. This made the ex vivo sorting of biot$\mathrm{mAb}$-coated DCs possible by use of anti-biot $\mathrm{mAb}$ coupled to magnetic beads. At $3 \mathrm{~h}$ postinjection, no TB10.4 presentation was detected with total low-density spleen cells recovered from mice injected with TB10.4-SA complexed to biot-control IgG. In contrast, positive anti-biot bead selected fractions from mice injected with TB10.4-SA complexed to biot-mAbs specific to CD11b or CD11c or to DCIR-2 or CD209 (Figure 2c) were able to stimulate the TB10.4-specific $1 \mathrm{H} 2$ T-cell hybridoma. In parallel, $\mathrm{BALB} / \mathrm{c}$ mice were injected intradermally (i.d.) with 50 pmol $(=1 \mu \mathrm{g})$ per mouse of TB10.4-SA complexed at a molar ratio of 1:1 to biot-control IgG or biot-mAbs specific to $\mathrm{CD} 205$ or CD207. At $3 \mathrm{~h}$ postinjection, positive fractions selected with biot-mAbs specific to CD205 or CD207 from the dermis were again able to stimulate $1 \mathrm{H} 2 \mathrm{~T}$ cells (Figure 2c). Positive and negative cell fractions from the spleen or dermis were able to present the synthetic TB10.4:74-88 peptide, showing the functional capacity of $\mathrm{Ag}$ presentation in all sorted cells (Figure 2d).

\section{ESX Ags delivery to $\boldsymbol{\beta}_{2}$-integrins or C-type lectins induces potent Th1 and Th17 responses}

We noticed that the TB10.4-SA alone induced some degrees of CD40, CD80, or CD86 upregulation (Supplementary Figure S2A online) and inflammatory cytokine and chemokine production by BM-DC (Supplementary Figure S2B online). However, this effect was additive to that of poly I:C. Of note, biot-anti-CD11b or -CD11c mAbs alone had no notable effect on DC maturation.
The potential of the ESX Ag delivery to DCs to trigger T-cell responses was then investigated in vivo with or without poly I:C as adjuvant, based on its well-established structural interaction with endosomal TLR3 or with cytosolic double-stranded RNA sensors, i.e., retinoic acid-inducible gene-I and melanoma differentiation-associated gene-5 RNA helicases, expressed by multiple cell types. ${ }^{14}$ Poly I:C also induces type-I IFNs, necessary to induce Th1 and CTL responses. ${ }^{15}$ DCs triggered via TLR3 also drive Th17 differentiation. ${ }^{16}$

This immunization strategy was first assessed subsequent to a single injection. C57BL/6 mouse groups $(n=3)$ immunized i.v. with $50 \mathrm{pmol}(=1 \mu \mathrm{g})$ of ESAT-6-SA, either without biot$\mathrm{mAb}$ or complexed to biot-control IgG, did not develop ESAT6-specific, MHC-II-restricted T-cell responses (Figure 3a). Immunization with ESAT-6-SA and biot-anti-CD11b mAb without poly I:C did not induce T-cell response. In contrast, mice immunized i.v. (Figure 3a) or subcutaneously (s.c.) (Supplementary Figure S3 online) with $50 \mathrm{pmol}(=1 \mu \mathrm{g})$ of ESAT-6-SA complexed to biot-anti-CD11b, -CD11c, or -CD205 mAbs with poly I:C-mounted strong T-cell responses, characterized by the production of IFN- $\gamma$ and IL-17 (Figure 3a,b), but not IL-5 (not shown). Delivery of ESAT-6 to CD207 induced weaker, but still marked IFN- $\gamma$ T-cell responses. ESAT-6 targeting to CD209 was not immunogenic, possibly due to the anti-inflammatory properties of CD209 ligation. ${ }^{17}$ When CpG (TLR-9 agonist) was used as an adjuvant together with ESAT-6-SA and biotanti-CD11b mAb, Th1-but not Th17-responses were also induced, yet at a lesser extent compared with poly I:C (Supplementary Figure S4A,B online).

Although the ESX-SA protein used at $1 \mu \mathrm{m}$ in vitro induced some degrees of DC maturation (Supplementary Figure S2 online), in vivo immunization with $50 \mathrm{pmol}$ per mouse of ESAT-6-SA delivered to CD11b, without an adjuvant, did not induce detectable T-cell responses (Figure 3a,b), suggesting that, due to the weak amounts of ESX-SA required to stimulate immune responses in vivo, these recombinant proteins-or their possible contaminants-did not reach sufficient concentration in the microenvironment of the targeted DCs to induce their maturation.

In this framework, Ag delivery to pDCs via BST-2 was not feasible, as poly I:C, which induces the production of type I/II IFN, ${ }^{18}$ induced BST-2 expression by a broad range of immune cells (Figure 3c) and loss of pDC specificity of BST-2 expression.

In conclusion, following a single immunization, Ag delivery to $\beta_{2}$-integrins or to CD205 was as efficient for T-cell priming, while CD207 targeting induced less intense T-cell responses and CD209 targeting did not initiate T-cell response. Compared with CD205, targeting $\beta_{2}$-integrins was more efficient at Th17 induction, while CD207 or CD209 targeting did not prime Th17.

Importantly, TB10.4-SA or CFP-10-SA delivered to CD11b or CD205 also induced Th1 responses, respectively, in BALB/c $\left(\mathrm{H}-2^{\mathrm{d}}\right)$ (Figure 4a) and $\mathrm{C} 3 \mathrm{H}\left(\mathrm{H}-2^{\mathrm{k}}\right)$ (Figure 4b) mice, 
a

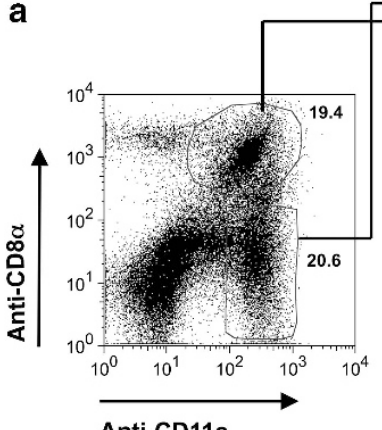

Anti-CD11c
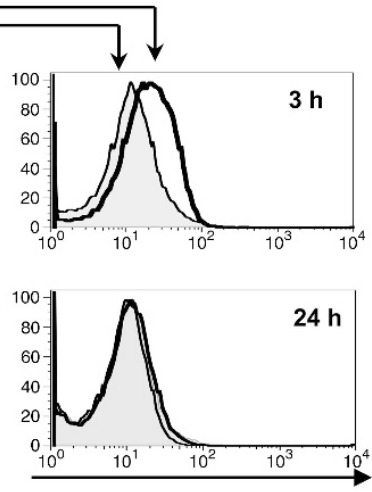

Anti-ESAT-6 b
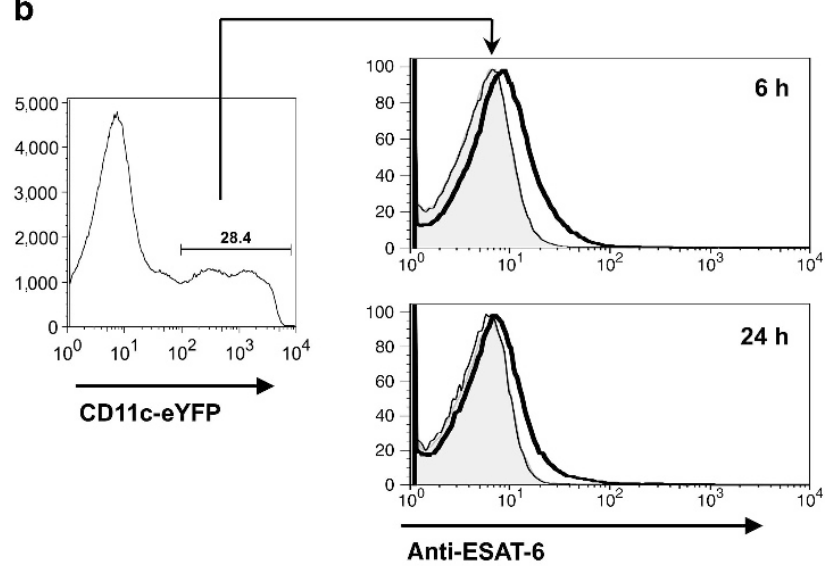

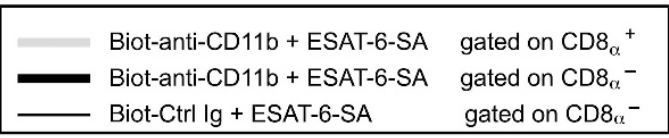

C

TB10.4-SA

injected with: Cell fraction

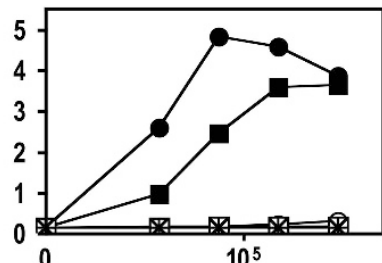

Biot-anti-CD11b $-{ }^{-}$CD11 ${ }^{+}$

Biot-anti-CD11b ${ }^{-} \mathrm{O}^{-} \mathrm{CD} 11 \mathrm{~b}^{-}$

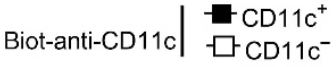

Biot-Ctrl Ig | * Tot. low-density cells

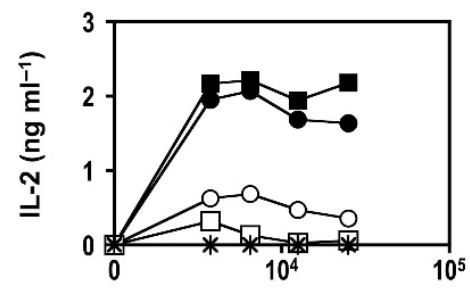

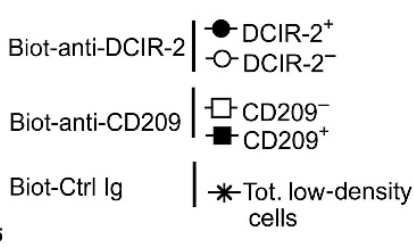
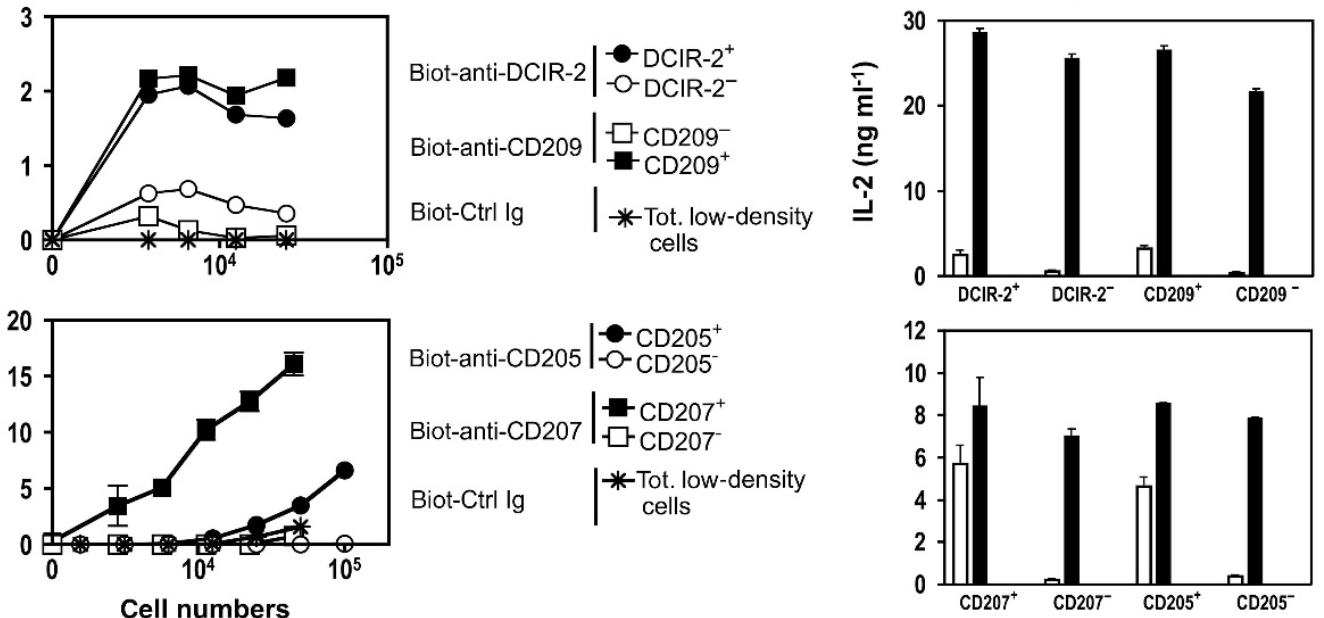

Figure 2 In vivo low-molecular weight proteins of the 6-kDa Early Secreted Antigenic Target (ESAT-6) protein family (ESX) antigen (Ag) presentation by the targeted dendritic cell (DC) subsets. (a) Tracking of ESAT-6 at the surface of spleen DCs of mice injected with ESAT-6-streptavidin (SA) complexed to biotin (biot)-anti-CD11b monoclonal Ab (mAb). C57BL/6 mice were injected intravenously (i.v.) with 500 pmol per mouse of ESAT-6-SA complexed at a molar ratio of 2:1 to biot-control (Ctrl) immunoglobulin G (IgG) or biot-anti-CD11b mAb in the presence of Poly(cytidylicinosinic) (Poly l:C). At different time points postinjection, low-density spleen cells were gated as $\mathrm{CD} 11 \mathrm{c}^{+} \mathrm{CD} 8_{\alpha}^{+}$or $\mathrm{CD} 11 \mathrm{c}^{+} \mathrm{CD} 8_{\alpha}^{-}$cells, and ESAT-6 surface signal was analyzed by anti-ESAT-6 mAb. (b) In vivo tracking of ESAT-6 at the surface of spleen DCs of mice injected with ESAT-6-SA complexed to biot-anti-CD11c mAb. CD11c-eYFP C57BL/6 mice were injected as in a with ESAT-6-SA complexed to biot-control IgG or biot-anti-CD11c mAb. Spleen low-density cells were gated as CD11c-eYFP cells, and ESAT-6 surface signal was analyzed. The results are representative of two independent experiments. (c, top and middle) Ex vivo presentation of TB10.4 by targeted DCs. BALB/c mice were injected subcutaneously (s.c.) with $50 \mathrm{pmol}$ per mouse of TB10.4-SA complexed at a molar ratio of 1:1 to mAbs specific to different DC surface receptors in the presence of Poly I:C. Spleen low-density cells were sorted $3 \mathrm{~h}$ postinjection by anti-biot mAb conjugated to magnetic beads and co-cultured with TB10.4-specific 1H2 T-cell hybridoma cells, and interleukin (IL)-2 levels were assessed by enzyme-linked immunosorbent assay (ELISA). (c, bottom) Ex vivo presentation of TB10.4 by dermal DCs. BALB/c mice were injected intradermally (i.d.) with 50 pmol per mouse of TB10.4-SA complexed to anti-CD205 or anti-CD207 biot-mAbs in the presence of Poly I:C. Dermal low-density cells were then sorted $3 \mathrm{~h}$ postinjection. Various numbers of cells from sorted fractions were co-cultured with $1 \mathrm{H} 2 \mathrm{~T}$-cell hybridomas. (d) The positive and negative fractions were tested for their capacity to present the synthetic TB10.4:74-88 peptide added in vitro by co-culture with 1H2 T-cell hybridoma cells to evaluate their functional capacity to present Ags to specific T-cell receptors (TCRs). The results are representative of two independent experiments. 

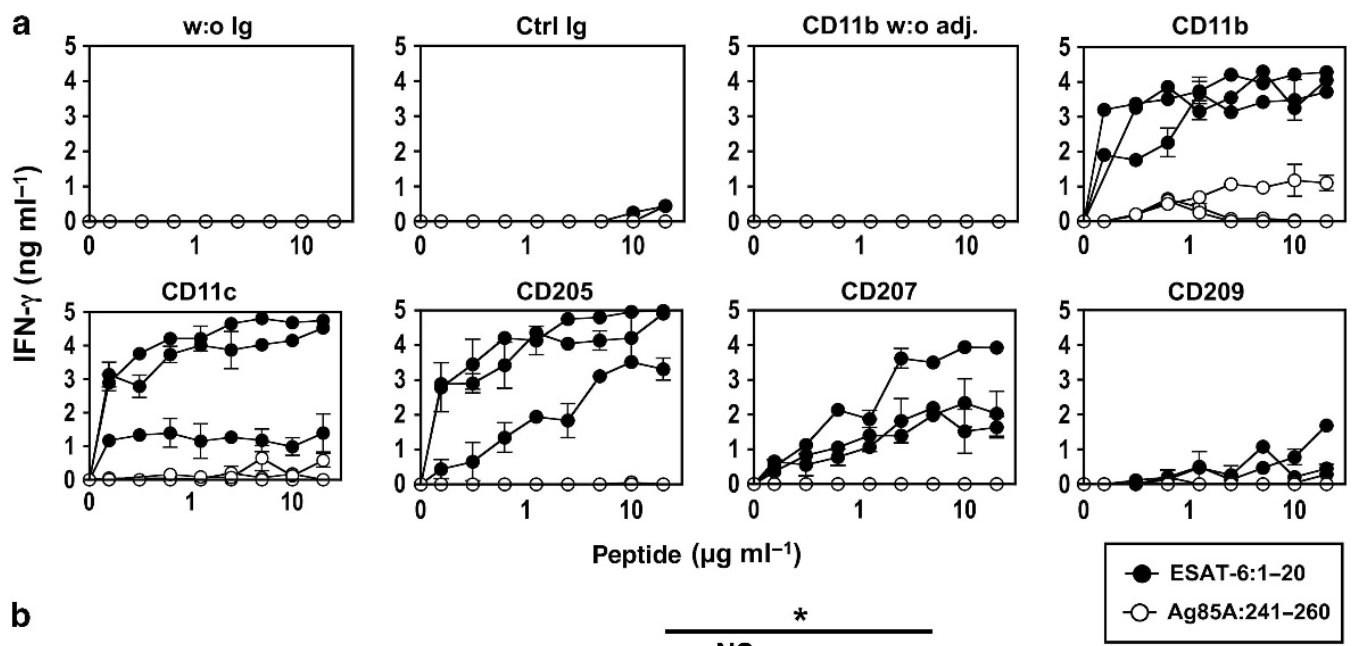

b

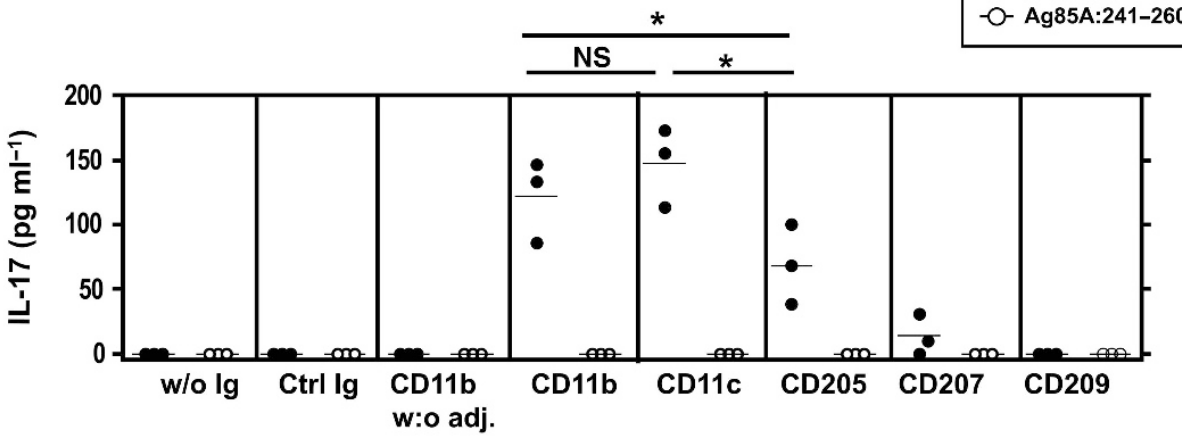

C
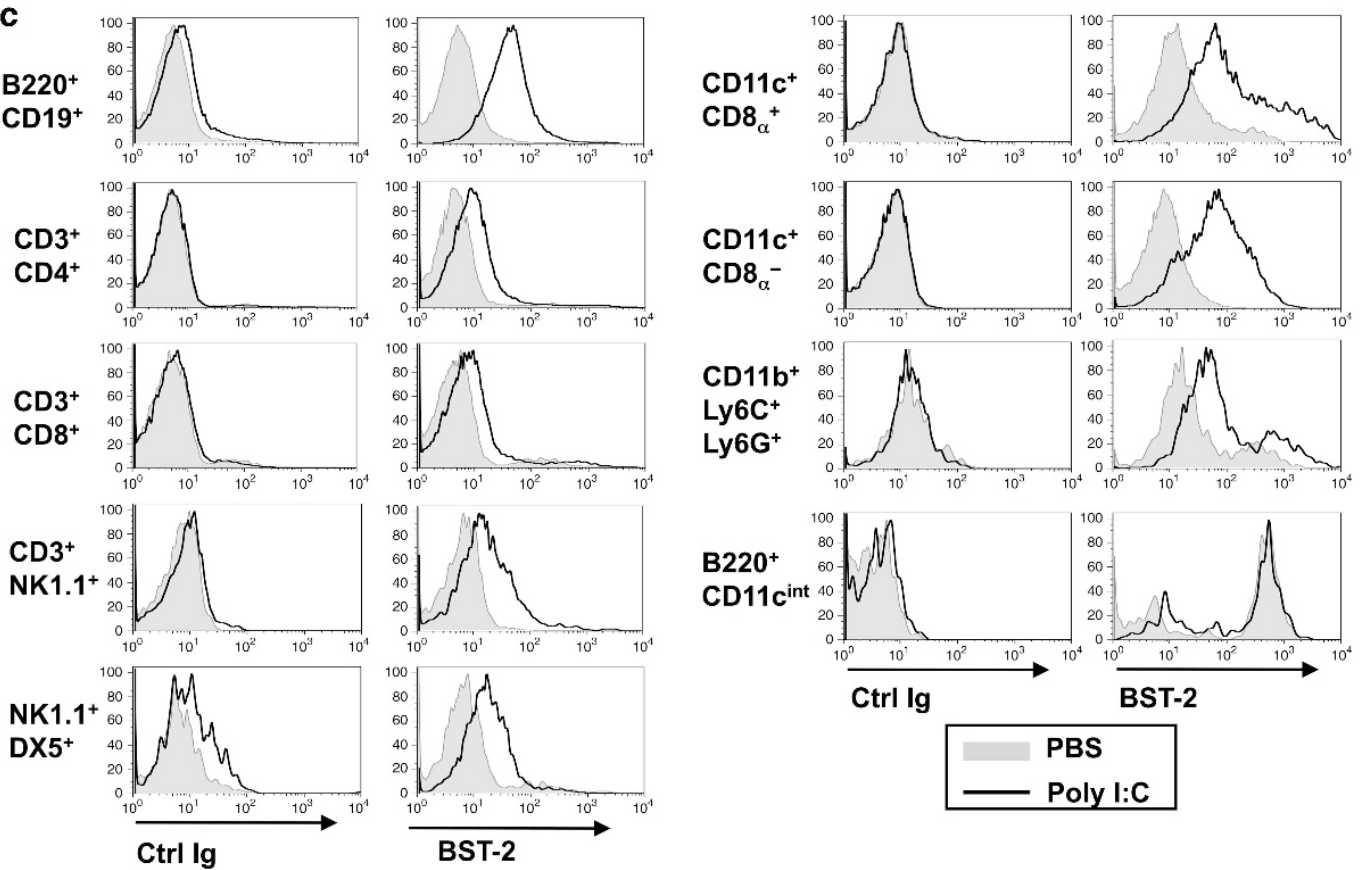

Figure 3 In vivo delivery of low-molecular weight proteins of the 6-kDa Early Secreted Antigenic Target (ESAT-6) protein family (ESX) antigens (Ags) to $\beta_{2}$-integrins or C-type lectins and induction of potent CD4 ${ }^{+}$T-cell responses. (a) Induction of CD4 ${ }^{+}$T-cell responses by ESAT-6 delivery to $\beta_{2}$-integrins or C-type lectins. C57BL/6 mice were immunized intravenously (i.v.) with 50 pmol per mouse of ESAT-6-streptavidin (SA) without biotin-immunoglobulin (biot-Ig) or complexed to biot-control (Ctrl) IgG or biot-monclonal antibodies (mAbs) specific to different $\beta_{2}$-integrins or C-type lectins in the presence of $25 \mu \mathrm{g}$ per mouse of Poly(cytidylic-inosinic) (Poly I:C). A control group received ESAT-6-SA complexed to biot-anti-CD11b in the absence of adjuvant. At day 11 postinjection, total splenocytes from three individual mice per group were stimulated in vitro with various concentrations of major histocompatibility complex (MHC)-II-restricted ESAT-6:1-20 peptide or Ag85A:241-260 as a negative control peptide. Interferon (IFN)- $\gamma$ responses were measured at $72 \mathrm{~h}$ and are shown as results from individual mice. (b) ESAT-6-specific interleukin (IL)-17 responses were measured in the supernatants of the cultures stimulated with $10 \mu \mathrm{g} \mathrm{ml}^{-1}$ of peptide. Horizontal bars indicate the mean values. The results are representative of at least two independent experiments. *Statistically significant, as determined by Student's $t$ test, $P<0.05$, NS = not significant. (c) Systemic injection of Poly I:C extends the BST-2 (bone marrow stromal cell Ag-2) expression to several adaptive and innate immune cell types other than plasmacytoid dendritic cells (DCs). C57BL/6 mice were injected i.v. with $25 \mu \mathrm{g}$ per mouse of Poly I:C. At $6 \mathrm{~h}$ postinjection, spleen innate and adaptive cells were stained and gated for different subsets, and the expression level of BST-2 was determined. The results are representative of at least two independent experiments. 
a

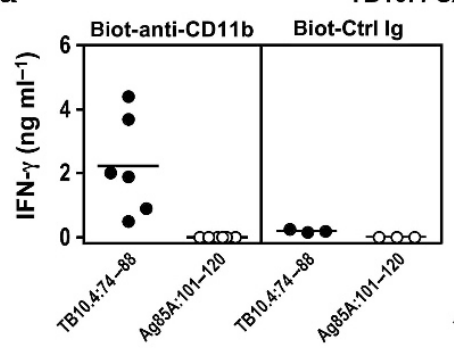

TB10.4-SA

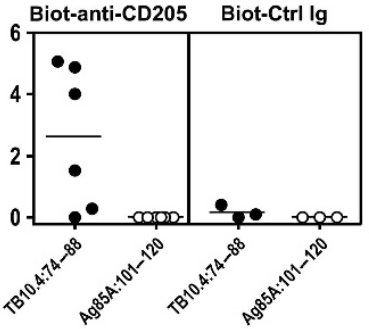

b CFP-10-SA

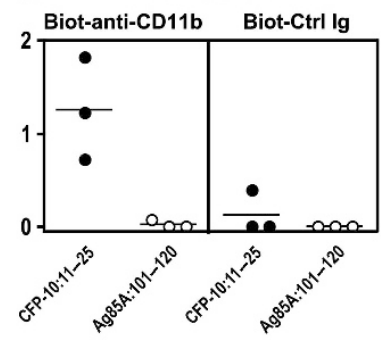

C
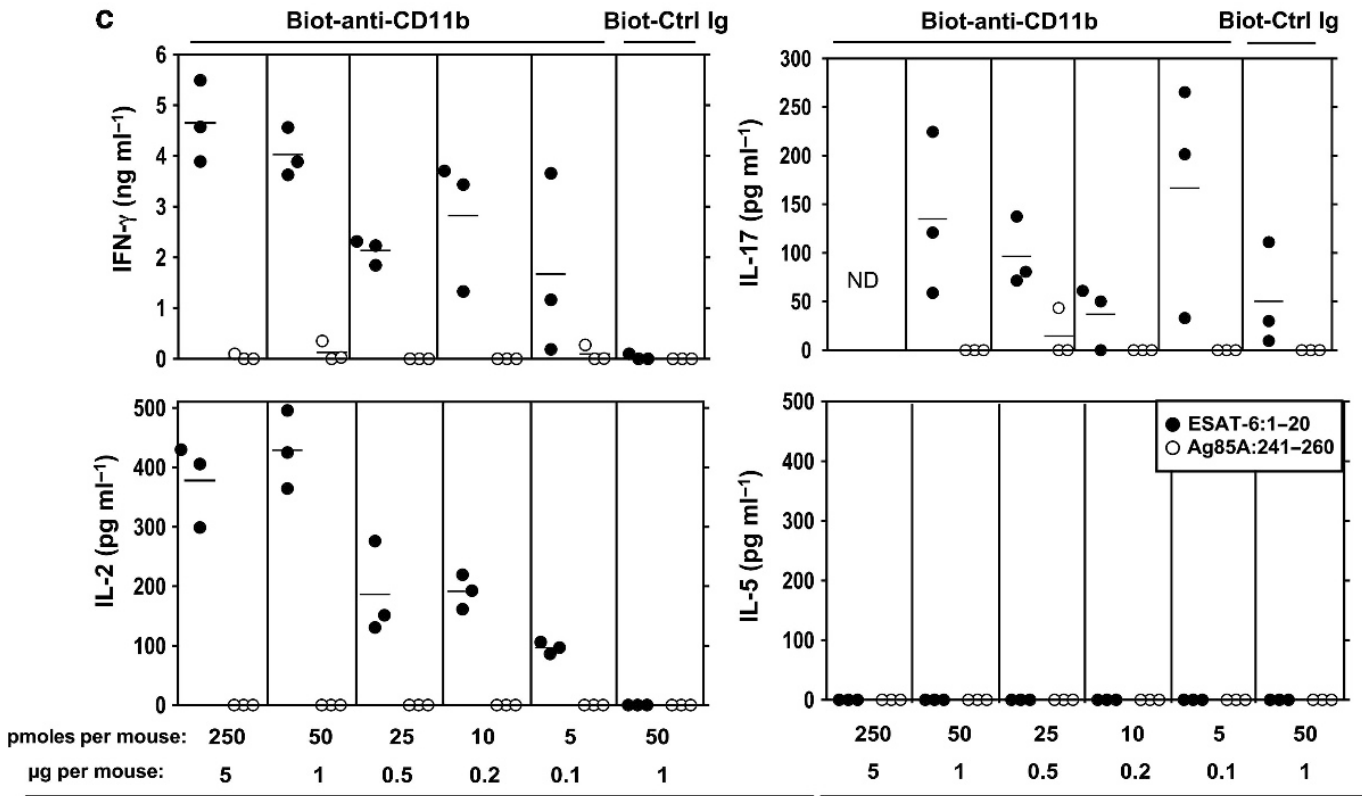

ESAT-6-SA

Figure 4 Induction of low-molecular weight proteins of the 6-kDa Early Secreted Antigenic Target (ESAT-6)- and CFP-10-specific T-helper (Th)1/Th17 $\mathrm{T}$-cell responses by antibody (Ab) delivery to $\mathrm{CD} 11 \mathrm{~b}^{+}$or $\mathrm{CD}_{20} 5^{+}$dendritic cells (DCs). (a, b) Induction of a CD4 ${ }^{+} \mathrm{T}_{\text {-cell response to TB10.4 or }}$ CFP-10 by the delivery of TB10.4 to CD11b or CD205 in BALB/c $\left(\mathrm{H}-2^{\mathrm{d}}\right)$ mice (a) or of CFP-10 to CD11b in C3H $\left(\mathrm{H}-2^{\mathrm{k}}\right) \mathrm{mice}(\mathbf{b})$. (c) C57BL/6 mice were immunized with various doses of ESAT-6-streptavidin (SA) complexed to biotin (biot)-anti-CD11b monoclonal Ab (mAb) at a molar ratio of 2:1 in the presence of Poly(cytidylic-inosinic) (Poly I:C). Interferon (IFN)- $\gamma$, interleukin (IL)-2, IL-17, and IL-5 responses were quantified at day 11 postinjection subsequent to in vitro stimulation of splenocytes with synthetic peptides containing major histocompatibility complex (MHC)-II-restricted T-cell epitopes. Ctrl, control; ND, not determined.

extending the feasibility of the strategy to various ESX Ags and MHC haplotypes and genetic backgrounds.

We also evaluated $\mathrm{T}$-cell responses after the administration of ESAT-6-SA complexed to biot-anti-CD11b mAb at different doses ranging from 250 to $5 \mathrm{pmol}(=5-0.1 \mu \mathrm{g})$ per mouse. The rational of CD11b targeting was its consistent efficiency in the previous experiments and the necessity to keep the targeted DC receptor constant to vary several other parameters. Only $50 \mathrm{pmol}(=1 \mu \mathrm{g})$ per mouse were enough to induce potent IFN- $\gamma$, IL-2, and IL-17, but not IL-5 responses at their maximal intensities (Figure 4c). As little as $5 \mathrm{pmol}(=0.1 \mu \mathrm{g})$ per mouse of ESAT-6-SA were still able to trigger Th1 and Th17 responses, showing the efficiency of the Ag delivery approach.

\section{Other features of the ESX Ag delivery}

The hypothesis that the biot-mAbs operate in vivo via $\mathrm{Fc}$ receptors (FcRs) rather than their specific DC surface ligands was negated by the observation that ESAT-6-SA complexed to biot-control IgG did not induce T-cell responses (Figure 3a,b). Moreover, $\mathrm{FcR} \gamma^{\circ}$ mice mounted lymphoproliferative and
IFN- $\gamma$ (Figure 5a,b) responses comparable to their wild-type counterparts after immunization with ESAT-6 delivery to CD11b.

Treg-mediated control of effector T cells has to be taken into account in immunization strategies. ${ }^{19,20}$ Lymphoproliferative, IFN- $\gamma$ and IL-2 (Figure 5c,d) responses induced by ESAT-6-SA delivery to CD11b were controlled by Tregs, as shown by their increase in anti-CD25 mAb-treated, i.e., Treg-attenuated mice, compared with their Treg-sufficient counterparts, treated with a control Ig isotype.

\section{Efficient boosting of BCG-induced T-cell responses by Ab-mediated delivery of ESX Ags to DCs}

Priming with BCG followed by boosting with potent subunit vaccines is considered the most promising $\mathrm{TB}$ vaccination strategy. ${ }^{8}$ We evaluated T-cell responses in BCG-primed mice boosted with ESX Ags delivered through different DC receptors. Given the increasing interest of C-type lectins in Ag delivery systems, ${ }^{21}$ we choose these DC receptors as target. $\mathrm{BALB} / \mathrm{c}$ mice, naïve or primed with $1 \times 10^{6}$ colony-forming 


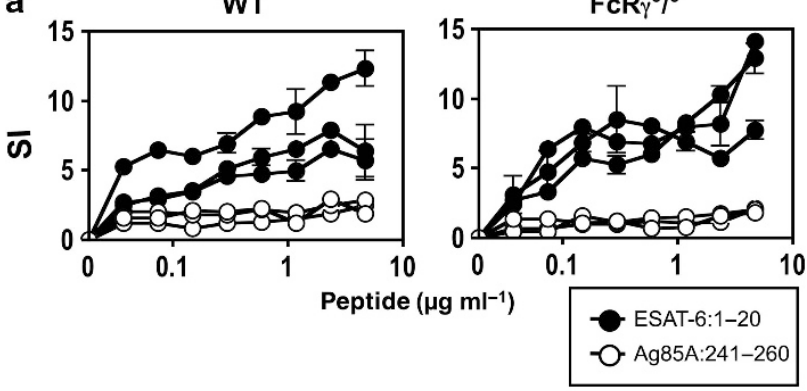

b

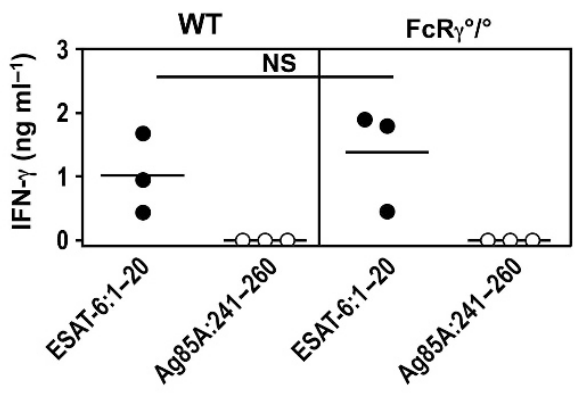

C

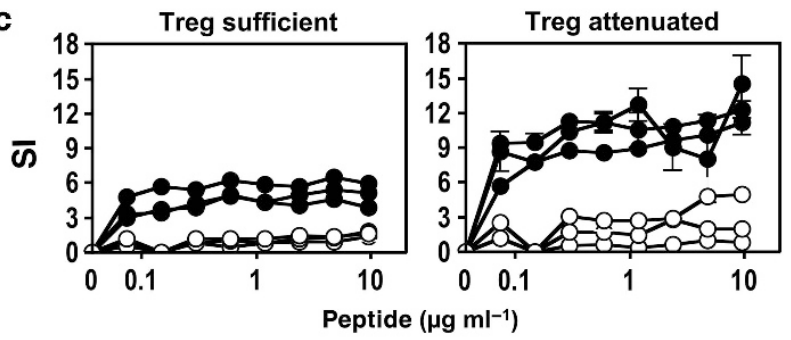

d
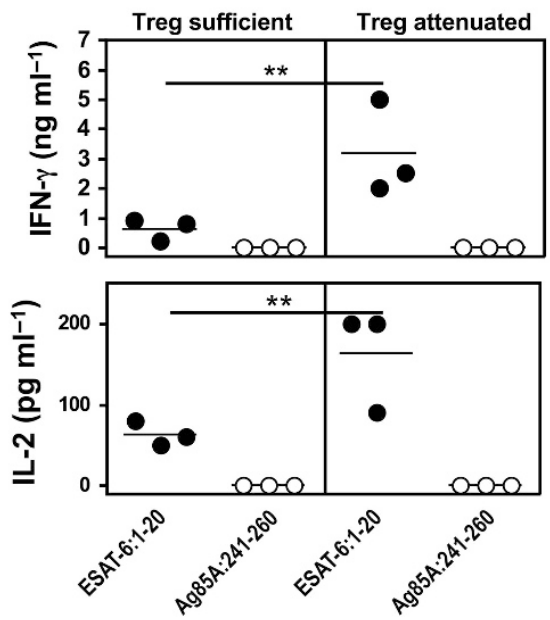

Figure 5 Induction of low-molecular weight proteins of the 6-kDa Early Secreted Antigenic Target (ESAT-6) protein family (ESX) antigen (Ag) CD4 ${ }^{+}$ $\mathrm{T}$-cell responses by antibody (Ab)-mediated delivery to dendritic cells (DCs) without interaction with $\mathrm{Fc}$ receptor (FcR). Control of the induced T-cell responses by Treg cells. (a) The proliferative response, expressed as stimulation index (SI) or (b) interferon (IFN)- $\gamma$ CD4 ${ }^{+}$T-cell responses of splenocytes from wild-type (WT) or FcR $\gamma^{\circ}$ C57BL/6 mice immunized intravenously (i.v.) with ESAT-streptavidin (SA) complexed to biotin (biot)-anti$\mathrm{CD} 11 \mathrm{~b}$ monoclonal $\mathrm{Ab}(\mathrm{mAb})$ following in vitro stimulation with $10 \mu \mathrm{g} \mathrm{ml}^{-1}$ of peptides. The results are from three individual mice per group, as determined at day 11 postimmunization. NS = not significant, as determined by Student's $t$-test. (c) Proliferative and (d) IFN- $\gamma$ or interleukin (IL)-2 responses in C57BL/6 mice treated with $1 \mathrm{mg}$ per mouse of control immunoglobulin (Ig) (Treg sufficient) or anti-CD25 (PC61) mAb (Treg attenuated) 2 days before immunization with ESAT-6-SA complexed to biot-anti-CD11b mAb in the presence of poly(cytidylic-inosinic) (poly I:C). Fc $\gamma \mathrm{R}$, Fc $\gamma$ receptor. unit per mouse of BCG on day 0 , were injected i.v. on days 14 and 21 with 50 pmol $(=1 \mu \mathrm{g})$ per mouse of TB10.4-SA complexed to different biot-mAbs. Two rounds of intranasal (i.n.) immunizations were performed based on the fact that numerous vaccination regimens require multiple injections. On day 28 , notable $\mathrm{CD} 4^{+}$T-cell IFN- $\gamma$ responses were detected in BCG-unprimed mice that were immunized with TB10.4 delivered to CD205, CD207, CD209, or DCIR-2, with the highest levels obtained with CD207 and DCIR-2 (Figure 6a). Such responses were comparably increased following boosting of BCG-primed mice with TB10.4-SA complexed to biot-mAbs specific to CD205, CD207, CD209, or DCIR-2, but not to the biot-control IgG.

Th17 responses were not detectable in BCG-unprimed $\mathrm{BALB} / \mathrm{c}$ mice immunized with TB10.4-SA complexed to different biot-mAbs (Figure 6b). In contrast, Th17 responses were induced in BCG-primed mice when TB10.4 was delivered to CD205 and, to a lesser extent, upon delivery through CD207, CD209, or DCIR-2. Determination of the reasons of the lack of Th17 responses in BCG-unprimed BALB/C mice immunized by TB10.4 delivery to CD205 and the presence of Th17 responses in C57BL/6 mice immunized by ESAT-6 delivery to CD205 (Figure 3b) will require more investigation in these two models with comparable immunization regimens.

In mice immunized with TB10.4 delivered to CD205, the levels of TB10.3/4:20-28-specific $\mathrm{CD}^{+}{ }^{+} \mathrm{T}$ splenocytes were low but detectable (Figure 6c), whereas in BCG-immunized mice, boosting with TB10.4 delivered to CD205 induced high frequencies of TB10.3/4:20-28-specific $\mathrm{CD} 8^{+} \mathrm{T}$ splenocytes, showing the ability of this vaccine strategy to induce CD8 ${ }^{+}$ T-cell responses.

\section{Mucosal immunization by TB10.4 delivery to lung CD205 ${ }^{+}$ DCs induces TB protection}

Having demonstrated the highly specific Ag delivery to splenic DC subsets and the efficiency of specific T-cell triggering upon i.v. immunization, we next transposed it to i.n. immunization to induce protective mucosal anti-mycobacterial immunity. In line with previous reports, ${ }^{22}$ we observed that CD205 is specifically expressed by all lung $\mathrm{CD} 11 \mathrm{c}^{+}$cells, and its expression level remained constant following i.n. poly I:C treatment (Figure 7a). Notably, the CD205 expression was not extended to other lung innate cells, i.e., $\mathrm{CD} 11 \mathrm{~b}^{+} \mathrm{F} 4 / 80^{+}$,

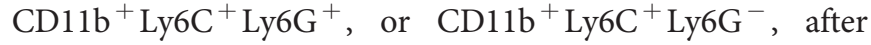
poly I:C treatment (not shown). We therefore evaluated whether CD205 represents an appropriate target for $\mathrm{Ag}$ delivery to lung DCs. BALB/c mice were immunized on days 0 and 9 with i.n. injections of $600 \mathrm{pmol}(=15 \mu \mathrm{g})$ per mouse of TB10.4-SA complexed at a molar ratio of 4:1 to biotanti-CD205 mAb. This immunization resulted in TB10.4specific $\mathrm{CD} 4{ }^{+}$T-cell IFN- $\gamma$ responses detectable at day 22 in the lungs (Figure $7 \mathbf{b}$ ).

To evaluate the protective potential of this immunization, $\mathrm{BALB} / \mathrm{c}$ mice were vaccinated following the same regimen, whereas control individuals were immunized s.c. on day 0 with 
a
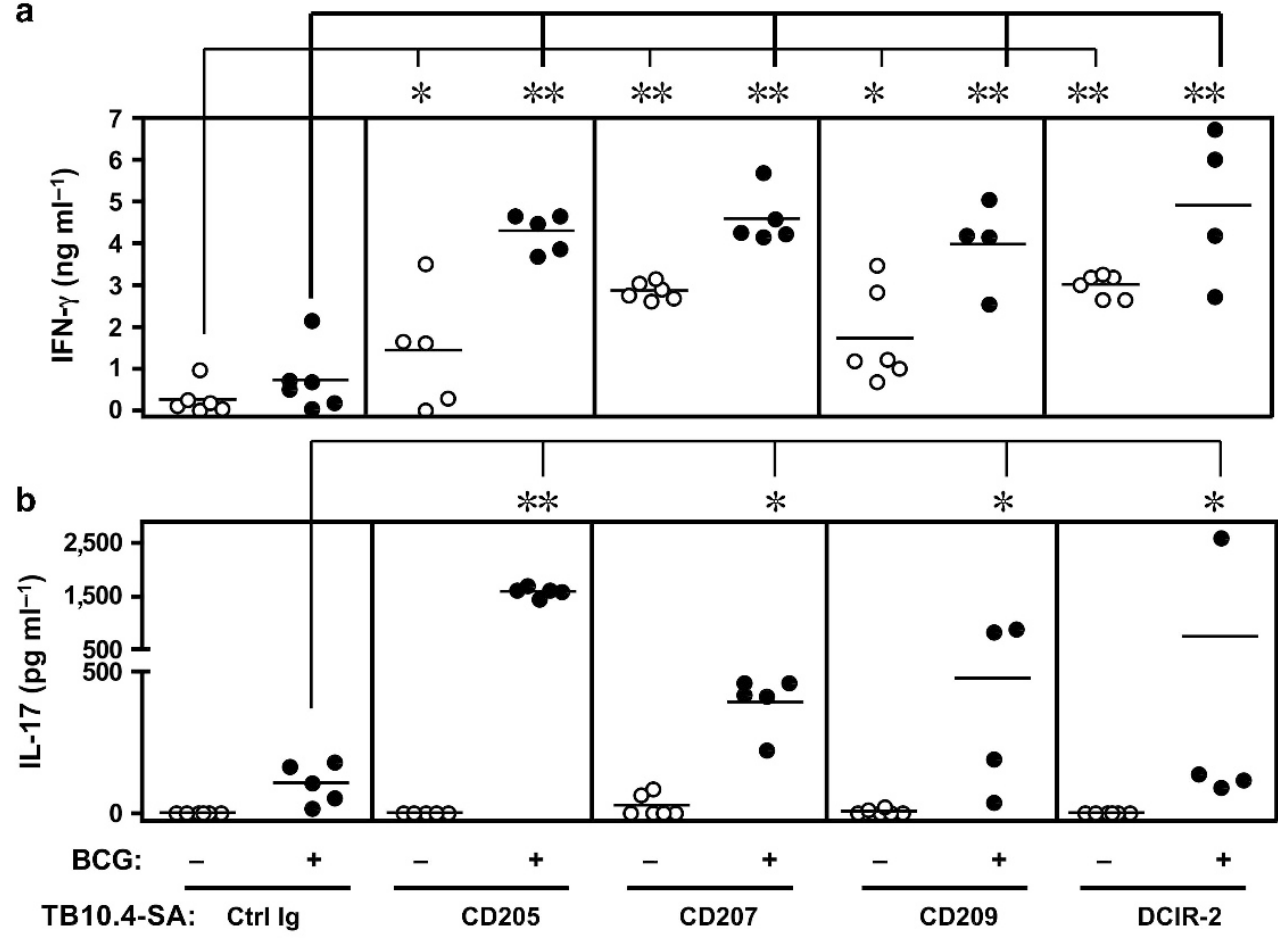

C Anti-CD44

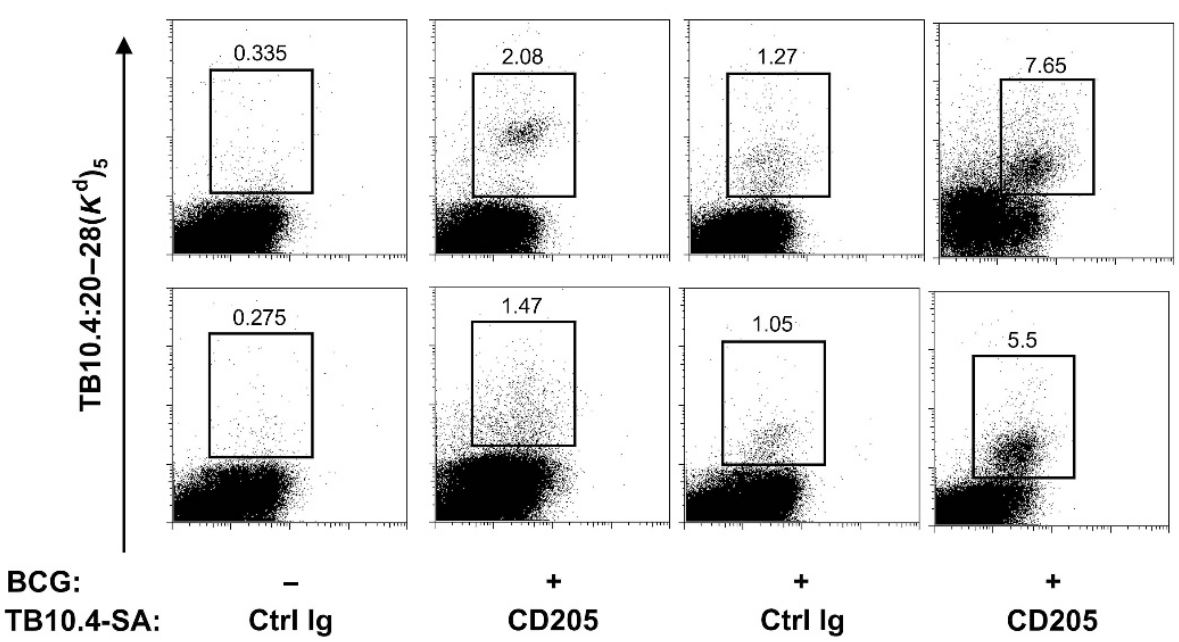

Figure 6 Efficient restimulation of Bacillus Calmette-Guérin (BCG)-induced memory responses by antibody (Ab) delivery of low-molecular weight proteins of the 6-kDa Early Secreted Antigenic Target (ESAT-6) protein family (ESX) antigen (Ag) to dendritic cell (DC) surface receptors. $\mathrm{BALB} / \mathrm{c}$ mice, naïve or primed subcutaneously (s.c.) with $1 \times 10^{6}$ colony-forming unit (CFU) of BCG at day 0 , were boosted twice at days 14 and 21 with $50 \mathrm{pmol}(=1 \mu \mathrm{g})$ per mouse of TB10.4-streptavidin (SA) complexed to biotin (biot)-control (Ctrl) immunoglobulin G (IgG) or biot-mAb specific to different C-type lectins at a molar ratio of 2:1 in the presence of poly(cytidylic-inosinic) (poly I:C). TB10.4-specific interferon (IFN)- $\gamma$ (a) or interleukin (IL)-17 (b) CD4 ${ }^{+} \mathrm{T}$-cell responses were studied at day 28 . * and ${ }^{* *}$ Statistically significant as determined by Student's $t$-test $(P<0.05$ and 0.005 , respectively). (c) $\mathrm{CD}^{+}$T-cell crosspriming in naïve or BCG-primed mice injected with TB10.4 delivered to CD205 as described in (a). CD8 ${ }^{+} \mathrm{T}^{-c e l l}$ responses to the $\mathrm{H}-2 \mathrm{~K}^{\mathrm{d}}$-restricted TB10.3/4:20-28 epitope, as detected by phycoerythrin (PE)-conjugated $\mathrm{H}-2 \mathrm{~K}^{\mathrm{d}}$ pentamer complexed to TB10.3/4:20-28 in viable $\mathrm{PI}^{-} \mathrm{CD} 8_{\alpha}^{+}$-gated splenocytes. Two representative individual mice are shown from two independent experiments. PI, propidium iodide.

BCG as a "gold standard" vaccine control to which new TB vaccine candidates are systematically compared. ${ }^{23}$ Mice were challenged with an aerosol of $M$. tuberculosis on day 22. At 1 month postinfection, a decreased mycobacterial burden was observed in the lungs from mice immunized with TB10.4 directed to airway CD205 ${ }^{+}$DCs compared with lungs from mice unvaccinated or injected with TB10.4-SA complexed to biot-control IgG. In parallel, a substantial decrease of mycobacterial dissemination to the spleen was observed (Figure 7c). In contrast, mice immunized by i.v. route with TB10.4-SA delivered to CD11b, CD205, or CD207 only displayed a weak protection and this did not reach 

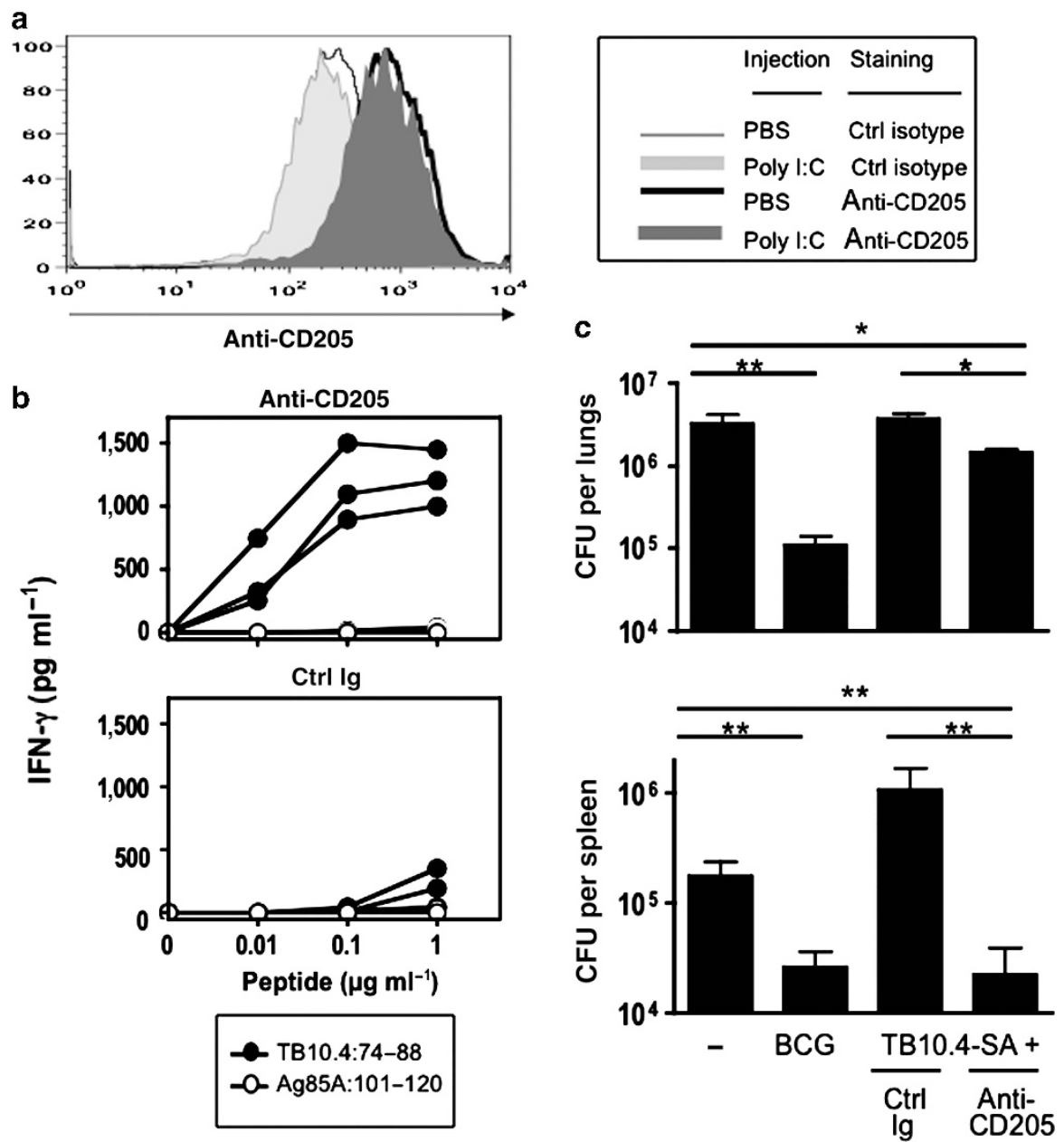

Figure 7 Induction of mucosal interferon (IFN)- $\gamma$ T-cell responses and lung protection by immunization with TB10.4-streptavidin (SA) delivered to airway CD205 ${ }^{+}$cells. (a) CD205 expression in lung CD11c ${ }^{+}$cells at steady state or 1 day after intranasal (i.n.) injection of phosphate-buffered saline (PBS) or $10 \mu \mathrm{g}$ per mouse poly(cytidylic-inosinic) (poly I:C). (b) BALB/c mice ( $n=3$ mice per group) were immunized i.n. with $15 \mu \mathrm{g}$ ( $=600$ pmol) per mouse of TB10.4-SA complexed to biotin (biot)-control (Ctrl) immunoglobulin G (IgG) or biot-anti-CD205 monoclonal antibody $(\mathrm{mAb})$ in the presence of poly I:C. Interferon (IFN)- $\gamma$ responses were quantified at day 14 postinjection after in vitro stimulation of lymphocytes enriched from the lungs in the presence of bone marrow (BM)-dendritic cells (DCs) loaded with TB10.4:74-88 or control peptide. The results from individual mice are shown. (c) Anti-M. tuberculosis protection conferred by delivery of TB10.4 to lung CD205 ${ }^{+}$cells. Mice were injected i.n. at days 0 and 9 with 600 pmol per mouse of TB10.4-SA complexed at a molar ratio of 4:1 to biot-control IgG or biot-anti-CD205-mAb. Control mice were immunized with Bacillus Calmette-Guérin (BCG). Mice were challenged on day 22 with M. tuberculosis H37Rv. Mycobacterial loads in lungs and spleen were determined at 1 month postchallenge. The results are representative of two independent experiments. ${ }^{*},{ }^{*}$ Statistically significant as determined by Mann-Whitney $t$-test $(P<0.05$ or $P<0.001$, respectively).

statistical significance (Supplementary Figure S5 online). This observation is in accordance with previous data showing that priming of mycobacteria-specific effector $\mathrm{T}$ cells in the local lung lymph nodes and their recruitment to the airway lumen at the time of pulmonary TB exposure are a more reliable correlate for protection than the presence of peripherally activated specific T cells. ${ }^{24,25}$

Thus, our results show that ESX Ag delivery to the lung $\mathrm{CD} 205^{+}$DCs by the technology described herein represents a promising step forward in the design of novel TB vaccine candidates.

\section{DISCUSSION}

Immunization based on the delivery of immunogens to DC subsets may provide new insights into the type of adaptive protective immunity. However, as the mechanisms governing functions of DC subsets are not thoroughly understood, it remains difficult to predict which DC subsets are the most appropriate targets to optimize protective immunity. Here, to compare the impact of targeting the different DC subsets on mycobacteria-specific protective immunity, we developed a versatile approach allowing the delivery of mycobacterial immunogens to different DC surface receptors. Our approach consists of the genetic fusion of mycobacterial immunogens from the ESX family to SA. The resulting Ag-SA fusion tetramers can then form stable complexes with targeting biot-mAbs specific for a range of receptors on DC surfaces. The Ag-SA:biot-mAb complexes can then deliver these immunogens into DCs through endocytosis with the selected surface receptors, thus enabling $\mathrm{Ag}$ processing and presentation and leading to the induction of adaptive immunity. 
This approach allowed efficient delivery of ESX-SA to selected DC subsets through $\beta_{2}$-integrins or C-type lectins. The ensuing efficient endocytosis of cell-bound Ag-SA:biot-mAb complexes was most likely due to the crosslinking of the targeted receptors by the biot-mAbs, leading to their capping together with the bound ESX-SA cargo. The ESX Ags were then processed and presented on MHC-II molecules to specific $\mathrm{T}$-cell receptors in an efficient manner. This Ag delivery was highly specific; only the ex vivo sorted DC subset targeted with ESX-SA complexed to the selected biot-mAbs displayed the bound ESX on their surfaces and were able to trigger specific T cells. We further demonstrated the efficient induction of ESXspecific Th1, Th17, and CD8 ${ }^{+}$T-cell responses when injected concomitantly with the adjuvant.

ESX Ags are short-length proteins, which do not possess multiple T-cell epitopes for different MHC haplotypes. Because of its strong I- $\mathrm{A}^{\mathrm{b}}$-restricted ESAT-6:1-20 epitope, ESAT-6 is highly immunogenic in $\mathrm{C} 57 \mathrm{BL} / 6\left(\mathrm{H}-2^{\mathrm{b}}\right)$ mice, $^{26}$ whereas TB10.4, with its strong $\mathrm{I}-\mathrm{A}^{\mathrm{d}}$-restricted TB10.4:74-88 and $\mathrm{K}^{\mathrm{d}}$-restricted TB10.4:20-28 T-cell epitopes, is much more immunogenic in $\mathrm{BALB} / \mathrm{c}\left(\mathrm{H}-2^{\mathrm{d}}\right)$ mice. $^{27}$ Therefore, the evaluation of the immunogenicity of these two ESX Ags necessitates the use of $\mathrm{H}-2^{\mathrm{b}}$ and $\mathrm{H}-2^{\mathrm{d}}$ strains.

With different biot-mAbs specific to $\beta_{2}$-integrins or C-type lectins, we directly compared the efficiencies of ESX Ag delivery to different DC subsets in the induction of T-cell responses. Remarkably, a single injection of only $1 \mu \mathrm{g}(=50 \mathrm{pmol})$ per mouse of ESX-SA complexed to biot-mAbs specific to CD11b, $\mathrm{CD} 11 \mathrm{c}$, or CD205 induced specific and highly sensitive Th1 and Th17 responses. ESX-SA complexed to biot-mAbs specific to CD207 induced less intense, yet still marked Th1 responses. The efficient induction of Th1 responses by ESX Ag targeting to $\beta_{2}$-integrins is in accordance with: (i) the substantial potential of other CD11b-targeting delivery vectors, such as the recombinant adenylate cyclase CyaA of Bordetella pertussis, in the induction of T-cell immunity ${ }^{27-30}$ and (ii) the notable $\mathrm{T}$-cell responses following $\mathrm{mAb}$-mediated OVA Ag targeting to CD11c. ${ }^{31}$ Among the C-type lectins evaluated here, CD205 was the most efficient target receptor for Ag-SA delivery to DCs and induction of Th1 and Th17 cells in primary and booster immunizations against ESX Ags. This integral transmembrane mannose receptor is intensely expressed by cortical thymic epithelium and DC subsets, including lung DCs. ${ }^{32}$ Moreover, compared with most other surface endocytic receptors, whose ligation induces endocytosis through early and more peripheral endosomes, CD205 is rapidly taken up after binding with carbohydrates, and its cytosolic domain mediates efficient endocytosis and recycling through the late endosomes and MHC-II-rich compartments. ${ }^{33}$ Furthermore, in addition to its capacity to shuttle Ags from the extracellular space into specialized MHC-II rich lysosomal compartments, CD205 is also able to introduce Ags to the MHC-I processing machinery. These properties likely account for the observed efficiency of crosspriming of TB10.4-specific CD8 ${ }^{+}$T-cell in BCG-primed mice that were boosted with TB10.4-SA targeted to CD205, but not other C-type lectins tested.
The significant efficiency of T-cell triggering following ESX-SA delivery to CD207 is in agreement with the significant T-cell responses subsequent to OVA Ag targeting through this C-type lectin. ${ }^{34}$ Importantly, CD207 mRNA is detectable in the mouse lungs and in the epithelium lining human airways. ${ }^{35}$ Directing of Ags for presentation by DCs through CD207 may thus be of interest for Ag delivery to lung DCs and induction of mucosal $\mathrm{T}$ cells close to the primary site of the mycobacterial infection.

In addition to the early production of IL-17 by lung T-cell receptor $\gamma \delta^{+} \mathrm{T}$ cells, ${ }^{36} \mathrm{CD} 4{ }^{+}$Th17 cells can be detected in mycobacteria-exposed mice and humans. ${ }^{37}$ However, the direct contribution of Th17 in the control of M. tuberculosis infection remains debatable. ${ }^{38,39}$ Mice immunized through delivery of ESX-SA to CD11b, CD11c, or CD205 developed significant Th17 responses. Notably, the DC receptor candidates that most significantly induced the Th17 responses also induced the highest Th1 responses. This is in accordance with the hypothesis that Th17 cells may facilitate the chemoattraction of Th1 cells. ${ }^{40}$

The strategy developed here enabled the induction of lung mucosal Th1 responses and reproducible anti-mycobacterial protection when used in i.n. immunization with TB10.4 directed to airway $\mathrm{CD} 205^{+}$DCs. These data provide proof of principle for directing mycobacterial Ags to airway DC subsets, which leads to the induction of mucosal immunity and protection against infection. Our results further demonstrate that the i.v. and i.n. routes of immunization differ in their ability to induce TB protection. Indeed, in accordance with previous data, ${ }^{25}$ our study clearly shows that the presence of specific $\mathrm{T}$ cells at the primary site of the mycobacterial infection, subsequent to i.n. mucosal immunization, rather than a strong systemic T-cell response, is required for the control of mycobacterial infection. Considering that several human counterparts of mouse DC subtypes have been identified and that targeted Ag delivery to endocytic C-type lectins at DC surface allows efficient Ag presentation in human, this vaccination strategy leads to new advances that increases our insight of the development of new human TB vaccines. The use of recombinant ESX Ags either alone or in fusion with other mycobacterial immunogens, formulated in appropriate adjuvants, constitutes a promising TB subunit vaccine strategy. ${ }^{41,42}$ Our approach should not to be considered as competing with the other subunit TB vaccines under development. Rather, it represents a potent complementary strategy that can now be evaluated for direct delivery of newly described Ags or combination of protective early and latency-associated mycobacterial $\mathrm{Ags}^{41}$ to lung DC and is expected to allow substantial reduction of the required dose of $\mathrm{Ag}$ and better control of the quality of the adaptive immunity.

\section{METHODS}

ESX-SA fusion proteins and peptides. The construction and purification of the Ag-SA fusion proteins have been described recently. ${ }^{12}$ Briefly, the open reading frames encoding ESX Ags ESAT-6, CFP-10, or TB10.4 were PCR-amplified with specific primers (Supplementary 
Table S1 online) and fused in frame to the $5^{\prime}$ end of a codon-optimized synthetic gene encoding residues 13-139 of SA from Streptomyces. Sequence-confirmed Ag-SA fusion proteins were produced as soluble Ag-SA tetramers as described. ${ }^{12}$ Lipopolysaccharide content in eluted proteins was reduced by passage through EndoTrap columns (Profos, Regensburg, Germany) to levels below 50 endotoxin unit (EU) of lipopolysaccharide per $\mathrm{mg}$ of protein, as determined by the Limulus Amebocyte Lysate assay (Lonza, Walkersville, MD).

The ESAT-6:1-20, CFP-10:11-25, TB10.4:74-88 peptides, containing MHC-II-restricted T-cell epitopes, and TB10.3/4:20-28 peptide, containing an MHC-I restricted T-cell epitope, were synthesized by NeoMPS (Strasbourg, France).

Biot-mAbs. mAbs specific to CD11b (M1/70.15.11.5.HL, rat $\left.\operatorname{IgG}_{2 \mathrm{~b}}\right)$, CD11c (N418, hamster IgG), DCIR-2 (33D1, rat IgG 2 ), MHC-II (I-A/ I-E) $\left(M 5 / 114.15 .2\right.$, rat $\left.\operatorname{IgG}_{2 \mathrm{~b}}\right)$, or the control Ig (R187, rat IgG) were biotinylated as described. ${ }^{12}$ Biot-anti-CD205 mAb (NLDC-145, rat $\mathrm{IgG}_{2 \mathrm{a}}$ ) was from Celldex Therapeutics (Needham, MA). Biot-mAbs specific to CD207 (eBioL31, rat IgG Iga $_{2}$ ), CD209 (LWC06, rat IgG Ia $_{2}$ ), or CD317 (BST-2) (eBio927, rat $\operatorname{IgG}_{2 \mathrm{~b}}$ ) were from eBioscience (San Diego, CA). Absence of endotoxin in the IgG preparations was verified by the Limulus Amebocyte Lysate assay.

Detection of ESAT- 6 binding to DC surface receptors. Conventional DCs or pDCs were generated as described ${ }^{43}$ and were incubated at $1 \times 10^{6}$ cells per well at $4^{\circ} \mathrm{C}$ with $1.5 \mu \mathrm{g} \mathrm{ml}^{-1}$ of biot-mAbs. Cells were then washed and incubated with $1 \mu \mathrm{m}$ of ESAT-6-SA for $1 \mathrm{~h}$ at $4^{\circ} \mathrm{C}$. The presence of ESAT-6-SA on DC surfaces was detected by cytofluorometry using the anti-ESAT-6 mAb (11G4; Antibody Shop, Gentofte, Denmark) labeled with fluoprotein $(\mathrm{FP})_{647 \mathrm{H}}$ (Interchim, Montluçon, France). Mean fluorescence intensity (MFI) reduction of the cell surface-bound ESAT-6-SA after endocytosis were calculated as $100-\left(\left(\mathrm{MFI}_{\text {biot-mAb }+ \text { ESAT-6-SA } 37^{\circ} \mathrm{C}}\right)-\left(\mathrm{MFI}\right.\right.$ biot-control IgG + ESAT-6-SA $\left.4^{\circ} \mathrm{C}\right) /$ $\left(\right.$ MFI biot-mAb + ESAT-6-SA $\left.4^{\circ} \mathrm{C}\right)-\left(\right.$ MFI biot-control IgG + ESAT-6-SA $\left.\left.4^{\circ} \mathrm{C}\right)\right) \times 100$.

In vivo binding of ESAT-6-SA to spleen DC subsets was studied after i.v. injection of ESAT-6-SA complexed with the biot-mAbs together with poly I:C. Low-density splenocytes ${ }^{44}$ were stained with a combination of DC-specific and $\mathrm{FP}_{647 \mathrm{H}}$-anti-ESAT- 6 mAbs for cytoflurometric analysis.

In vitro and ex vivo Ag presentation assays. $\mathrm{BM}-\mathrm{M} \phi$ or $-\mathrm{DCs}$ were plated at $1 \times 10^{5}$ cells per well and incubated with $1.5 \mu \mathrm{g} \mathrm{ml}^{-1}$ of biot$\mathrm{mAbs}$ for $30 \mathrm{~min}$ at $4^{\circ} \mathrm{C}$. Cells were then washed and incubated with various concentrations of ESX-SA. Cells were then co-cultured overnight with $1 \times 10^{5}$ cells per well of I-A ${ }^{\mathrm{b}}$-restricted, ESAT-6:1-20specific $(\mathrm{NB} 11)^{45}$ or I-A ${ }^{\mathrm{d}}$-restricted, TB10.4-6:74-88-specific $(1 \mathrm{H} 2)^{46}$ $\mathrm{T}$-cell hybridomas. The efficiency of $\mathrm{Ag}$ presentation to $\mathrm{T}$-cell hybridomas was evaluated by ELISA (enzyme-linked immunosorbent assay) IL-2 assay in the co-culture supernatants.

For ex vivo Ag presentation assays, mice were injected i.v. or i.d. with TB10.4-SA complexed to biot-mAbs with poly I:C. At $3 \mathrm{~h}$ postinjection, low-density cells from the spleen or the ear were stained with antibiot $\mathrm{mAb}$ coupled to magnetic microbeads and AutoMacsPro (Miltenyi Biotec, Paris, France) for positive selection of targeted cells. Various numbers of sorted cells were co-cultured with antiTB10.4:74-88 T-cell hybridomas, and IL-2 was assessed in the supernatants after $24 \mathrm{~h}$.

Mice and immunization. Female $\mathrm{BALB} / \mathrm{c}, \mathrm{C} 57 \mathrm{BL} / 6$, or $\mathrm{C} 3 \mathrm{H}$ mice (Charles Rivers, Arbresle, France) were immunized at 6-12 weeks old. Ag-SA and biot-mAbs were complexed at the indicated doses at $4{ }^{\circ} \mathrm{C}$ for $1 \mathrm{~h}$ and were injected i.v. at $200 \mu \mathrm{l}$ per mouse. BCG (Pasteur $1173 \mathrm{P} 2$ ) was injected s.c. at the base of the tail. $\mathrm{C} 57 \mathrm{BL} / 6 \mathrm{Fc} \gamma \mathrm{R}^{\circ}$ mice, deficient for the activating Fc $\gamma$ RI, III, and IV were provided by Pierre Bruhns and Marc Daeron (Institut Pasteur, Paris, France). C57BL/6 CD11c-eYFP mice were provided by Orchidée Filipe-Santos and Philippe Bousso (Institut Pasteur). Treg attenuation was performed by an intraperitoneal injection of $1 \mathrm{mg}$ per mouse of anti-CD25 mAb (PC61) or control IgG at 2 days before immunization. Animal studies were approved by the Institut Pasteur Safety Committee, the National Ethics Committee, in accordance with the European guidelines.

T-cell assays. CD4 ${ }^{+} \mathrm{T}$-cell assays were performed on splenocytes stimulated in vitro with various concentrations of synthetic peptides containing MHC-II-restricted epitopes. Culture supernatants were assessed at $24 \mathrm{~h}$ for the presence of IL- 2 and at $72 \mathrm{~h}$ for IL-5, IFN- $\gamma$, and IL-17A as described. ${ }^{47}$

CD8 ${ }^{+}$T-cell responses were evaluated in splenocytes, stimulated in vitro with $10 \mu \mathrm{g} \mathrm{ml}^{-1}$ of TB10.3/4:20-28 peptide. ${ }^{48}$ At day 6, specific $\mathrm{CD}^{+}{ }^{+} \mathrm{T}$ lymphocytes were detected by a phycoerythrinconjugated pentamer of $\mathrm{H}-2 \mathrm{~K}^{\mathrm{d}}$ complexed to TB10.3/4:20-28 (Proimmune, Oxford, UK) in the presence of FITC-anti-CD $8_{\alpha}$ (53-6.7) and allophycocyanin-anti-CD44 (Ly-24) (IM7) mAbs from $\mathrm{BD} /$ PharMingen (Le Pont de Claix, France). Cells were acquired on a Cyan cytometer (Beckman Coulter, Grenoble, France) and analyzed by FlowJo program (Treestar, OR).

Protection against $\boldsymbol{M}$. tuberculosis. BALB/c mice were anesthetized as described ${ }^{46}$ and were injected i.n. with 600 pmol $(=15 \mu \mathrm{g})$ per mouse of TB10.4-SA complexed to biot-control IgG or -anti-CD205$\mathrm{mAb}$ and poly I:C or were immunized s.c. with $1 \times 10^{4}$ colony-forming unit per mouse of BCG. Mice were challenged with $\approx 100$ colonyforming unit per mouse of $M$. tuberculosis $\mathrm{H} 37 \mathrm{Rv}$ via aerosol and mycobacterial loads were determined at 1 month postchallenge, as detailed previously. ${ }^{19}$

SUPPLEMENTARY MATERIAL is linked to the online version of the paper at http://www.nature.com/mi

\section{ACKNOWLEDGEMENTS}

This work was supported by grants from Agence National de la Recherche (ANR 2010-EMMA-008-01), the Ligue Nationale Contre le Cancer (Equipe Labellisée 2011), and the European Union's Seventh Framework Program (FP7/2007-2013) under Grant Agreement No.: 280873 ADITEC to C.L., O.S., and P.S. were supported by grants KAN200520702 and M200201213 and the Research Plan RVO:61388971. C.U. was a recipient of a Weissman International Internship Program Grant. The authors gratefully acknowledge P. Bruhns, M. Daeron, O. Filipe-Santos, and P. Bousso for providing various strains of mice and $\mathrm{E}$. Maranghi for excellent technical assistance for animal care and the aerosol challenge with $\mathrm{M}$. tuberculosis in the A3 animal facilities at Pasteur Institute.

\section{DISCLOSURE}

The authors declared no conflict of interest.

c 2013 Society for Mucosal Immunology

\section{REFERENCES}

1. Reis, e \& Sousa, C. Dendritic cells in a mature age. Nat. Rev. Immunol. 6, 476-483 (2006).

2. Bedoui, S. et al. Cross-presentation of viral and self antigens by skinderived CD103 + dendritic cells. Nat. Immunol. 10, 488-495 (2009).

3. de Heer, H.J., Hammad, H., Kool, M. \& Lambrecht, B.N. Dendritic cell subsets and immune regulation in the lung. Semin. Immunol. 17, 295-303 (2005).

4. Villadangos, J.A. \& Schnorrer, P. Intrinsic and cooperative antigenpresenting functions of dendritic-cell subsets in vivo. Nat. Rev. Immunol. 7, 543-555 (2007).

5. Steinman, R.M. Dendritic cells in vivo: a key target for a new vaccine science. Immunity 29, 319-324 (2008).

6. Bonifaz, L., Bonnyay, D., Mahnke, K., Rivera, M., Nussenzweig, M.C. \& Steinman, R.M. Efficient targeting of protein antigen to the dendritic cell receptor DEC-205 in the steady state leads to antigen presentation on 
major histocompatibility complex class I products and peripheral CD8 + T cell tolerance. J. Exp. Med. 196, 1627-1638 (2002).

7. Trumpfheller, C. et al. Intensified and protective CD4 + T cell immunity in mice with anti-dendritic cell HIV gag fusion antibody vaccine. J. Exp. Med. 203, 607-617 (2006).

8. Kaufmann, S.H., Baumann, S. \& Nasser Eddine, A. Exploiting immunology and molecular genetics for rational vaccine design against tuberculosis. Int. J. Tuberc. Lung Dis. 10, 1068-1079 (2006).

9. Brodin, P., Rosenkrands, I., Andersen, P., Cole, S.T. \& Brosch, R. ESAT-6 proteins: protective antigens and virulence factors?. Trends Microbiol. 12, 500-508 (2004).

10. Pym, A.S. et al. Recombinant BCG exporting ESAT-6 confers enhanced protection against tuberculosis. Nat. Med. 9, 533-539 (2003).

11. Simeone, R., Bottai, D. \& Brosch, R. ESX/type VII secretion systems and their role in host-pathogen interaction. Curr. Opin. Microbiol. 12, 4-10 (2009).

12. Stanek, O., Linhartova, I., Majlessi, L., Leclerc, C. \& Sebo, P. Complexes of streptavidin-fused antigens with biotinylated antibodies targeting receptors on dendritic cell surface: a novel tool for induction of specific T-cell immune responses. Mol. Biotechnol. 51, 221-232 (2012).

13. Howarth, M. et al. A monovalent streptavidin with a single femtomolar biotin binding site. Nat. Methods 3, 267-273 (2006).

14. Kawai, T. \& Akira, S. Toll-like receptor and RIG-I-like receptor signaling. Ann. N Y Acad. Sci. 1143, 1-20 (2008).

15. Hervas-Stubbs, S., Olivier, A., Boisgerault, F., Thieblemont, N. \& Leclerc, C. TLR3 ligand stimulates fully functional memory CD8 $+T$ cells in the absence of CD4 + T-cell help. Blood 109, 5318-5326 (2007).

16. Veldhoen, M., Hocking, R.J., Atkins, C.J., Locksley, R.M. \& Stockinger, B. TGFbeta in the context of an inflammatory cytokine milieu supports de novo differentiation of IL-17-producing T cells. Immunity 24, 179-189 (2006).

17. Geijtenbeek, T.B. \& Gringhuis, S.I. Signalling through C-type lectin receptors: shaping immune responses. Nat. Rev. Immunol. 9, 465-479 (2009).

18. Blasius, A.L., Giurisato, E., Cella, M., Schreiber, R.D., Shaw, A.S. \& Colonna, M. Bone marrow stromal cell antigen 2 is a specific marker of type I IFN-producing cells in the naive mouse, but a promiscuous cell surface antigen following IFN stimulation. J. Immunol. 177, 3260-3265 (2006).

19. Jaron, B., Maranghi, E., Leclerc, C. \& Majlessi, L. Effect of attenuation of Treg during BCG immunization on anti-mycobacterial Th1 responses and protection against Mycobacterium tuberculosis. PLoS One 3 , e28332008).

20. Olivier, A., Sainz-Perez, A., Dong, H., Sparwasser, T., Majlessi, L. \& Leclerc, C. The adjuvant effect of TLR agonists on CD4 + effector T cells is under the indirect control of regulatory T cells. Eur. J. Immunol. 41, 2303-2313 (2011).

21. Unger, W.W. \& van Kooyk, Y. 'Dressed for success' C-type lectin receptors for the delivery of glyco-vaccines to dendritic cells. Curr. Opin. Immunol. 23 , 131-137 (2011).

22. Lagranderie, M. et al. Dendritic cells recruited to the lung shortly after intranasal delivery of Mycobacterium bovis BCG drive the primary immune response towards a type 1 cytokine production. Immunology 108 352-364 (2003).

23. Izzo, A., Brandt, L., Lasco, T., Kipnis, A.P. \& Orme, I. NIH pre-clinical screening program: overview and current status. Tuberculosis (Edinb) 85 , 25-28 (2005).

24. Santosuosso, M. et al. Mucosal luminal manipulation of T cell geography switches on protective efficacy by otherwise ineffective parenteral genetic immunization. J. Immunol. 178, 2387-2395 (2007).

25. Jeyanathan, M., Heriazon, A. \& Xing, Z. Airway luminal Tcells: a newcomer on the stage of TB vaccination strategies. Trends Immunol. 31, 247-252 (2010).

26. Brandt, L., Oettinger, T., Holm, A., Andersen, A.B. \& Andersen, P. Key epitopes on the ESAT- 6 antigen recognized in mice during the recall of protective immunity to Mycobacterium tuberculosis. J. Immunol 157 3527-3533 (1996).
27. Hervas-Stubbs, S. et al. High frequency of $\mathrm{CD} 4^{+} \mathrm{T}$ cells specific for the TB10.4 protein correlates with protection against Mycobacterium tuberculosis infection. Infect. Immun. 74, 3396-3407 (2006).

28. Guermonprez, P. et al. The adenylate cyclase toxin of Bordetella pertussis binds to target cells via the alpha(M)beta(2) integrin (CD11b/CD18). J. Exp. Med. 193, 1035-1044 (2001).

29. Majlessi, L. et al. An increase in antimycobacterial Th1-cell responses by prime-boost protocols of immunization does not enhance protection against tuberculosis. Infect. Immun. 74, 2128-2137 (2006).

30. Preville, X., Ladant, D., Timmerman, B. \& Leclerc, C. Eradication of established tumors by vaccination with recombinant Bordetella pertussis adenylate cyclase carrying the human papillomavirus 16 E7 oncoprotein. Cancer Res. 65, 641-649 (2005).

31. Kurts, C. CD11c: not merely a murine DC marker, but also a useful vaccination target. Eur. J. Immunol. 38, 2072-2075 (2008).

32. Shrimpton, R.E., Butler, M., Morel, A.S., Eren, E., Hue, S.S. \& Ritter, M.A. CD205 (DEC-205): a recognition receptor for apoptotic and necrotic self. Mol. Immunol. 46, 1229-1239 (2009).

33. Jiang, W. et al. The receptor DEC-205 expressed by dendritic cells and thymic epithelial cells is involved in antigen processing. Nature $\mathbf{3 7 5}$, 151-155 (1995).

34. Idoyaga, J. et al. Cutting edge: langerin/CD207 receptor on dendritic cells mediates efficient antigen presentation on $\mathrm{MHC} \mathrm{I}$ and II products in vivo. J. Immunol. 180, 3647-3650 (2008).

35. Valladeau, J. et al. Identification of mouse langerin/CD207 in Langerhans cells and some dendritic cells of lymphoid tissues. J. Immunol. 168, 782-792 (2002).

36. Lockhart, E., Green, A.M. \& Flynn, J.L. IL-17 production is dominated by gammadelta $\mathrm{T}$ cells rather than CD4 T cells during Mycobacterium tuberculosis infection. J. Immunol. 177, 4662-4669 (2006).

37. Umemura, M. et al. IL-17-mediated regulation of innate and acquired immune response against pulmonary Mycobacterium bovis bacille Calmette-Guerin infection. J. Immunol. 178, 3786-3796 (2007).

38. Scriba, T.J. et al. Distinct, specific IL-17- and IL-22-producing CD4 + Tcell subsets contribute to the human anti-mycobacterial immune response. J. Immunol. 180, 1962-1970 (2008).

39. Chackerian, A.A. et al. Neutralization or absence of the interleukin-23 pathway does not compromise immunity to mycobacterial infection. Infect. Immun. 74, 6092-6099 (2006).

40. Khader, S.A. et al. IL-23 and IL-17 in the establishment of protective pulmonary $\mathrm{CD} 4+\mathrm{T}$ cell responses after vaccination and during Mycobacterium tuberculosis challenge. Nat. Immunol. 8, 369-377 (2007).

41. Aagaard, C. et al. A multistage tuberculosis vaccine that confers efficient protection before and after exposure. Nat. Med. 17, 189-194 (2011).

42. van Dissel, J.T. et al. Ag85B-ESAT-6 adjuvanted with IC31 promotes strong and long-lived Mycobacterium tuberculosis specific T cell responses in naive human volunteers. Vaccine 28, 3571-3581 (2010).

43. Mouries, J., Moron, G., Schlecht, G., Escriou, N., Dadaglio, G. \& Leclerc, C. Plasmacytoid dendritic cells efficiently cross-prime naive T cells in vivo after TLR activation. Blood 112, 3713-3722 (2008).

44. Majlessi, L. et al. Influence of ESAT-6 secretion system 1 (RD1) of Mycobacterium tuberculosis on the interaction between mycobacteria and the host immune system. J. Immunol. 174, 3570-3579 (2005).

45. Frigui, W. et al. Control of $M$. tuberculosis ESAT-6 secretion and specific T cell recognition by PhoP. PLoS Pathog. 4, e332008).

46. Sayes, F. et al. Strong immunogenicity and cross-reactivity of Mycobacterium tuberculosis ESX-5 type VII secretion-encoded PE-PPE proteins predicts vaccine potential. Cell Host Mic. 11, 352-363 (2012).

47. Majlessi, L. et al. Inhibition of phagosome maturation by mycobacteria does not interfere with presentation of mycobacterial antigens by $\mathrm{MHC}$ molecules. J. Immunol. 179, 1825-1833 (2007).

48. Majlessi, L., Rojas, M.J., Brodin, P. \& Leclerc, C. CD8 ${ }^{+}-$T-cell responses of Mycobacterium-infected mice to a newly identified major histocompatibility complex class I-restricted epitope shared by proteins of the ESAT-6 family. Infect. Immun. 71, 7173-7177 (2003). 